FSUJ-TPI-01/07

CGPG-01/12-1

\title{
Poisson Geometry in Constrained Systems
}

\author{
Martin Bojowald ${ }^{a}$ 五 and Thomas Strobl ${ }^{b}$ \\ ${ }^{a}$ Center for Gravitational Physics and Geometry, Department of Physics, \\ The Pennsylvania State University, University Park, PA 16802, USA \\ ${ }^{b}$ Institut für Theoretische Physik, Universität Jena, D-07743 Jena, Germany
}

\begin{abstract}
Associated to a constrained system with closed constraint algebra there are two Poisson manifolds $P$ and $Q$ forming a symplectic dual pair with respect to the original, unconstrained phase space: $P$ is the image of the constraint map (equipped with the algebra of constraints) and $Q$ the Poisson quotient with respect to the orbits generated by the constraints (the orbit space is assumed to be a manifold). We provide sufficient conditions so that the reduced phase space of the constrained system may be identified with a symplectic leaf of $Q$. By these methods, a second class constrained system with closed algebra is reformulated as an abelian first class system in an extended phase space.

While any Poisson manifold $(P, \Pi)$ has a symplectic realization (Karasev, Weinstein 87 ), it does not always permit a leafwise symplectic embedding into a symplectic manifold $(M, \omega)$. For regular $P$, it is seen that such an embedding exists, iff the characteristic form-class of $\Pi$, a certain element of the third relative cohomology of $P$, vanishes. A tubular neighborhood of the constraint surface of a general second class constrained system equipped with the Dirac bracket provides a physical example for such an embedding into the original symplectic manifold. In contrast, a leafwise symplectic embedding of e.g. (the maximal regular part of) a Poisson Lie manifold associated to a compact, semisimple Lie algebra does not exist.
\end{abstract}

*e-mail address: bojowald@gravity.phys.psu.edu

${ }^{\dagger}$ e-mail address: Thomas.Strobletpi.uni-jena.de 


\section{Contents}

\begin{tabular}{llr}
\hline 1 & Introduction & 2
\end{tabular}

2 Preliminaries-Different types of constraints 5

\begin{tabular}{|lll}
\hline 3 & Poisson geometry from closed constraint algebras & 9
\end{tabular}

3.1 Symplectic dual pairs and related morphisms . . . . . . . . . . . . . . . . . 10

3.2 Second class constraints . . . . . . . . . . . . . . . . . . . 13

3.3 The general case $\ldots \ldots \ldots$. . . . . . . . . . . . . . . . . . . . . 19

3.4 Transforming second class constraints to a first class system . . . . . . . . 23

$3.4 .1(M, \bar{\omega})$ as graph of the constraint map . . . . . . . . . . . . 24

3.4 .2 Extension by a Whitney sum . . . . . . . . . . . . . . . . . 25

3.4.3 $\quad$ Possible application and generalization . . . . . . . . . . . . . . . 26

4 Dirac brackets and leaf-symplectic embeddings of Poisson manifolds 28

4.1 Dirac bracket . . . . . . . . . . . . . . . . . . . . . . . . . . 28

4.2 Closed constraint algebra revisited . . . . . . . . . . . . . . . . . . 31

4.3 Compatible presymplectic forms on neighborhoods of regular leaves . . . . 32

4.4 Leafwise symplectic embeddings of Poisson manifolds . . . . . . . . . . . . 36

\section{Introduction}

Within constrained Hamiltonian mechanics one is used to the concept of presymplectic manifolds, manifolds equipped with a closed 2-form. Pulling back the symplectic form $\omega$ from the original phase space $M$ to the constraint surface $C$, the resulting 2 -form $\omega_{C}$ is still closed, but in general no longer nondegenerate. The constraints (functions on $M$ characterizing the constraint surface $C$ as joint preimage of zero) are of 'second class' [1], iff $\omega_{C}$ happens to be nondegenerate; $\left(C, \omega_{C}\right)$ is then the reduced phase space of the theory. Otherwise, there are vector fields in the kernel of $\omega_{C}$, which are integrable as a consequence of the Frobenius theorem; their orbits are the 'gauge transformations' of the theory. Taking the factor space, provided well-defined, yields the reduced (or physical) phase space in this more general case.

Recently there has been increasing interest in Poisson manifolds, in part because of its relation to deformation quantization (cf. e.g. [2, 3, 4]) and the interplay of String Theory with noncommutative gauge theories (cf. e.g. [5, 6]).

Poisson manifolds are a generalization of symplectic manifolds in a way dual to (but different from) the one of presymplectic manifolds: Instead of defining a symplectic manifold through the existence of a nondegenerate 2-form $\omega=\frac{1}{2} \omega_{i j} \mathrm{~d} x^{i} \wedge \mathrm{d} x^{j}$, closed due to Jacobi, one could as well define it by its (negative) inverse, i.e. by a nondegenerate bivector field $\Pi=\frac{1}{2} \Pi^{i j} \partial_{i} \wedge \partial_{j}$, where $\Pi^{i j}=-\left(\omega^{-1}\right)^{i j} \equiv\left\{x^{i}, x^{j}\right\}$, the fundamental 
Poisson brackets between local coordinates. Now the Jacobi identity takes the form $[\Pi, \Pi] \equiv-\Pi^{i j}{ }_{s} \Pi^{s k} \partial_{i} \wedge \partial_{j} \wedge \partial_{k}=0$. While presymplectic manifolds result from giving up the nondegeneracy of $\omega_{i j}$ (keeping $\mathrm{d} \omega=0$ ), Poisson manifolds result from giving up nondegeneracy of $\Pi^{i j}$ (keeping $[\Pi, \Pi]=0$ ). In the degenerate (nonsymplectic) Poisson case, Hamiltonian vector fields $\left.v_{f}:=-\mathrm{d} f\right\lrcorner \Pi \equiv\{\cdot, f\}$ do no longer span the full tangent space $T_{x} P$ at any $x \in P$. Still, the respective distribution is integrable (since $\left[v_{f}, v_{g}\right]=v_{\{g, f\}}$ due to Jacobi, i.e. due to $[\Pi, \Pi]=0$ ), generating a foliation (stratification) of $P$ into symplectic leaves.

In this paper we show that also the notion of Poisson manifolds plays some role within the physical scenario of constrained Hamiltonian systems. We show how (also nonsymplectic) Poisson manifolds arise naturally in this context and how they are related to the respective (pre)symplectic manifolds of the system.

The first instance, where Poisson manifolds show up, is in the case that the Poisson algebra of the constraint functions defines a closed Poisson subalgebra. As pointed out recently [7], such a constrained system does not only give rise to a presymplectic manifold (the constraint surface $C$ embedded in the original symplectic manifold) but is equally naturally associated to a Poisson manifold $P, P$ being the image of the original phase space $M$ under the constraint map, endowed with the Poisson algebra of these constraints. In fact, invoking theorems from the mathematics literature, any Poisson manifold $P$ or any presymplectic manifold $C$ (though not any pair $(P, C)$ ) can be regarded as arising in this manner (cf. Theorem 1 and 8 below).

Since the constraints form a closed algebra, their Hamiltonian vector fields define an integrable distribution (on all of $M$, not just on $C$ as one is used to from a general first class constrained system). Assume the quotient of $M$ with respect to these 'generalized gauge orbits' gives a well-defined, differentiable manifold $Q$. Then also $Q$ can be endowed with a natural Poisson bracket; essentially it is just the original Poisson algebra of 'gauge invariant' functions on $M$.

In Sec. 3 of this paper we refine this construction. In particular, we show that $P$ and $Q$ form a so called symplectic dual pair with respect to the original phase space $M$ and specify conditions (cf. Theorem 3, Corollary 2', and Theorem 1 below) such that the reduced phase space $R$, of primary physical interest, may be identified with a symplectic leaf of $Q$.

For a closed first class constrained system (i.e. the origin in $P$ is a symplectic leaf) this implies that we may reverse the two steps in the reduction process: Instead of first restricting to a submanifold (which is presymplectic) and then taking the factor space with respect to the gauge orbits, we may as well first take a factor space with respect to generalized gauge orbits, leading to the Poisson manifold $Q$, and only then take the restriction (in this case to a particular symplectic leaf).

Moreover, by these means also a closed second class constrained system may be viewed

\footnotetext{
${ }^{1}$ The reason for the somewhat conventional minus sign in front of the matrix inverse to $\omega_{i j}$ in the definition of $\Pi^{i j}$ will be commented on in Footnote 5 .

${ }^{2}$ In the context of physics, constraints are usually globally defined functions on $M$ and then only any region of $P$ homeomorphic to a subset of $\mathbb{R}^{d}, d=\operatorname{dim}(P)$, can be obtained. For most of our considerations, however, we can relax this condition on the constraints.
} 
similarly to a (general) first class constrained system. This will be made more explicit in Sec. 3.4 by embedding the original, unconstrained phase space $M$ into some extended phase space $\widetilde{M}$, where for any second class constraint function in $M$ one gets a first class constraint function in $\widetilde{M}$. We will show that upon an appropriate extension, one can manage to have the first class constraints to Poisson commute. This may constitute a significant simplification for the quantization of the original system.

There is also another instance where Poisson manifolds are used in the context of constrained systems. In the case of second class constraints $\Phi^{\alpha} \approx 0$ (not necessarily closed under taking Poisson brackets), it was Dirac himself who introduced a modified Poisson bracket on the original phase space, the Dirac bracket. This bracket has the feature that the constraints $\Phi^{\alpha}(x)$ are its Casimir functions and, essentially, that restricting to the constraint surface commutes with taking Poisson brackets. In other words, one defines a new Poisson bivector $\Pi_{D}$ such that one of its symplectic leaves coincides (as a symplectic manifold) with the reduced phase space $\Phi^{\alpha}=0$ of the theory. In fact, such a relation holds even for a whole neighborhood of leaves: The slightly deformed constraint surfaces $\Phi^{\alpha}=c^{\alpha}$, for constants $c^{\alpha}$ out of some interval containing zero, endowed with the symplectic form inherited from the embedding original phase space $(M, \omega)$, are symplectic leaves of $\Pi_{D}$.

In general $\Pi_{D}$ is defined only in some tubular neighborhood $S$ of the constraint surface $C$. What is the relation between $(M, \omega)$ and $\left(S, \Pi_{D}\right)$ ? Clearly, the restriction of the identity map on $M$ to $S$ is not a Poisson map since the respective Poisson brackets coincide only for particular functions. On the other hand, the embedding map from $S$ to $M$ is leafwise symplectic (or leaf-symplectic), i.e. restricted to any leaf of $\Pi_{D}$, the map is symplectic.

In Sec. 4 we consider the question whether a given Poisson manifold $\left(P, \Pi_{P}\right)$ admits a leaf-symplectic embedding into some symplectic manifold. Locally and around a regular point $x$ of $P$ such a map exists always: In a neighborhood $N$ of $x$ one may choose CasimirDarboux coordinates $\left(q^{\alpha}, p_{\beta}, C^{I}\right), I=1, \ldots, k$ where $k$ is the corank of $\Pi$ at $x$, for which the given bivector has the form $\Pi_{P}=\frac{\partial}{\partial q^{\alpha}} \wedge \frac{\partial}{\partial p_{\alpha}}$ [8]. The respective embedding phase space may be chosen as $\left(N \times \mathbb{R}^{k}, \mathrm{~d} p_{\alpha} \wedge \mathrm{d} q^{\alpha}+\mathrm{d} P_{I} \wedge \mathrm{d} C^{I}\right)$, where $P_{I}$ are linear coordinates in $\mathbb{R}^{k}$ and the embedding corresponds to fixing these 'Casimir momenta' to some value.

Globally, however, there can be obstructions which may be characterized by a closed 3 -form on $P$, the so called form-class [9] of the bivector $\Pi_{P}$. Considered as an element of the third relative cohomology of a regular Poisson manifold $\left(P, \Pi_{P}\right)$, its vanishing is necessary and sufficient for the existence of a leaf-symplectic embedding (cf. Proposition 11 below). The condition on the form-class may, furthermore, be cast into the form of descent equations, familiar to physicists from the analysis of anomalies (cf, e.g., [10]) and, more recently, also from the cohomological deformation of action functionals [11. In this manner we will find, e.g., that a family of coadjoint orbits of a compact, semisimple Lie algebra (viewed as Lie Poisson manifold) does not permit a leaf-symplectic embedding. On the other hand, any regularly foliated Poisson manifold whose leaves have trivial second cohomology does so.

\footnotetext{
${ }^{3} \mathrm{~A}$ function $C$ is called a Casimir function of a Poisson tensor $\Pi$ if $\Pi^{i j} \partial_{j} C=0$.
} 
Thus while there always exists a surjective Poisson map from a symplectic manifold to any given Poisson manifold [12, 13] (i.e., a so called symplectic realization, cf. Theorem 1 below), and likewise always a coisotropic embedding of (regular) presymplectic manifolds into a symplectic manifold [14, 15] (Theorem 2 below), a leaf-symplectic embedding of a given Poisson manifold exists only in particular cases.

Both relations of (pre)symplectic and Poisson manifolds studied in this paper have physical applications: The information in the symplectic dual pairs, and in particular the reformulation of a second class constrained system with closed algebra as an abelian first class system, can be used, e.g., in a path integral quantization, the prime example possibly being some Yang-Mills gauge theory where the constraint algebra is spoiled by an anomaly (cf. also [16, 17, 18] for related work). The existence of leaf-symplectic maps for a regular Poisson manifold leads to solutions [19, 20] of the associated Poisson Sigma model [21, 22] on a Riemann surface.

Before describing the new results we set the stage in Sec. 2, recalling the basic definitions of different types of submanifolds in a symplectic manifold, in particular the definitions of first and second class constraint surfaces going back to Dirac. On this occasion we also suggest generalizations of these notions to submanifolds of presymplectic and Poisson manifolds (Def. 2).

\section{Preliminaries - Different types of constraints}

We start with some general remarks on constrained systems. Let us consider a phase space, i.e. a symplectic manifold, $(M, \omega)$ in which we single out a submanifold $C$, called the constraint surface. We assume that this submanifold can be characterized as the intersection of the zero level set of $\left\{\Phi^{\alpha} \in C^{\infty}(M), \alpha=1, \ldots, d\right\}$, the constraint functions or simply the constraints. We further assume that the constraints are regular and irreducible, which means that $\bigwedge_{\alpha=1}^{d} \mathrm{~d} \Phi^{\alpha}$ does not vanish on $C$. The Hamiltonian of the system Poisson commutes with all the $\Phi^{\alpha}$, i.e. the set of $\Phi^{\alpha} \mathrm{S}$ is already the total set of constraintswe are not interested in a splitting into "primary" and "secondary" constraints [1] as it arises when starting from different Lagrangian systems, eventually leading to the same constrained Hamiltonian system.

We will, however, use the notion of first class and second class constraints:

Definition $1 A$ constraint function $\Phi^{\alpha}$ of a constrained system $C \hookrightarrow(M, \omega)$ is of the first (second) class, if its Hamiltonian vector field $v^{\alpha} \equiv\left\{\cdot, \Phi^{\alpha}\right\}$ is (is nowhere) tangent to $C$. The full set of constraint functions $\left\{\Phi^{\alpha}\right\}$, such that $C=\bigcap_{\alpha}\left(\Phi^{\alpha}\right)^{-1}(0)$, is of the first (second) class, if each single constraint function $\Phi^{\alpha}$ is of the first (second) class.

In other words, $\Phi^{\alpha}$ is of first (second) class, iff $v^{\alpha}$ lies (does not lie) in $\iota_{*} T C$ for all points on the constraint surface $C \subset M, \iota: C \rightarrow M$ denoting the respective embedding map.

In general a constraint will be neither of first nor second class, but of 'mixed type', and a splitting of constraints, characterizing a given constraint surface $C \subset M$, into first and second class constraints can be achieved only locally. 
The definition as given above is readily seen to reproduce the one given by Dirac [1] (use $\left.\left\{\Phi^{\alpha}, \Phi^{\beta}\right\}\right|_{C} \equiv v^{\beta}\left(\Phi^{\alpha}\right)$ or cf. Proposition 1 below): A system of constraints $\left\{\Phi^{\alpha}\right\}$ is of the first class, iff $\left.\left\{\Phi^{\alpha}, \Phi^{\beta}\right\}\right|_{C}=0 \forall \alpha, \beta$, and of the second class, iff $\left.\operatorname{det}\left\{\Phi^{\alpha}, \Phi^{\beta}\right\}\right|_{C} \neq 0$.

In the more mathematically inclined literature, the above two types of constraint surfaces are characterized in a different way, without any reference to the constraint functions. First note that the pullback bundle $\iota^{*} T M$ (or $\left.T M\right|_{C}$ ) is a symplectic vector bundle [23||t over $\mathrm{C}$ and $\iota_{*} T C$ (or simply $T C$ ) a subbundle thereof. This subbundle induces a symplectically orthogonal subbundle

$$
(T C)^{\perp}:=\left\{\left.v \in T M\right|_{C}: \omega(v, w)=0 \text { for all } w \in T C\right\}
$$

which is related to the annihilator of $T C$ in $M$ (or the "conormal bundle" of $C$ ),

$$
\operatorname{Ann}_{M}(T C)=\left\{\left.\alpha \in T^{*} M\right|_{C}: \alpha(v)=0 \text { for all } v \in T C\right\},
$$

in the following way. Denoting the map from a vector space $V$ to its dual $V^{*}$ induced by a bilinear form $B$ on $V$ as $B^{\sharp}, v \mapsto B(v, \cdot)$, the symplectic form $\omega \in \Omega^{2}(M)$ defines a bijection $\omega^{\sharp}: T M \rightarrow T^{*} M$. Denote by $\Pi^{\sharp}: T^{*} M \rightarrow T M$ the transpose inverse map, $\Pi^{\sharp}:=\left(\omega^{\sharp}\right)^{-1, T} \equiv-\left(\omega^{\sharp}\right)^{-1}$, which implicitly defines a bivector field $\Pi$. We now find $\Pi^{\sharp}\left(\operatorname{Ann}_{M}(T C)\right)=(T C)^{\perp}$ or $\operatorname{Ann}_{M}(T C)=\omega^{\sharp}\left((T C)^{\perp}\right)$ : The inclusion is established by noting that $\alpha \in \operatorname{Ann}_{M}(T C)$ implies $\omega\left(\Pi^{\sharp} \alpha, w\right)=-\alpha(w)=0$ for any $w \in T C$, while, vice versa, $\omega(v, w) \equiv \omega^{\sharp} v(w)=0$ for all $w \in T C$ implies $\omega^{\sharp}(T C)^{\perp} \subset \operatorname{Ann}_{M}(T C)$.

Clearly, $\operatorname{Ann}_{M}(T C)=\prec \mathrm{d} \Phi^{\alpha} \succ$ since $\mathrm{d} \Phi^{\alpha}(w)=w\left(\Phi^{\alpha}\right)$ vanishes for any tangent vector $w \in T C$. With $\left.v^{\alpha}=-\mathrm{d} \Phi^{\alpha}\right\lrcorner \Pi \equiv-\Pi^{\sharp} \mathrm{d} \Phi^{\alpha}$ we similarly recognize $(T C)^{\perp}$ as the span of the Hamiltonian vector fields generated by the constraints: $(T C)^{\perp}=\prec v^{\alpha} \succ$.

We now have several equivalent characterizations of first and second class constrained systems:

${ }^{4}$ Let us call a vector bundle endowed with a differentiable field of bilinear antisymmetric forms of its fibers a presymplectic vector bundle. If this bilinear form is nondegenerate, the bundle is symplectic [23]. Note that both the tangent bundle $T M$ of a presymplectic manifold $(M, \omega)$ and the cotangent bundle $T^{*} M$ of a Poisson manifold $(M, \Pi)$ are presymplectic vector bundles over $M$ in this terminology. In this context it is worthwhile mentioning a possible unification of the two apparently different generalizations of symplectic manifolds in terms of so called Dirac structures [24, 25. Here one starts from $T M \oplus T^{*} M$, endowed with the canonical symmetric bilinear form from the pairing and looks for certain maximally isotropic subbundles. (By definition a subbundle in a vector bundle equipped with a nondegenerate symmetric or antisymmetric bilinear form $\kappa$ on its fibers is isotropic if all of its sections are mutually orthogonal with respect to $\kappa$ ). Although it may be worthwhile to explore the considerations of the present paper within this more general framework of Dirac structures, we will not attempt to do so here.

${ }^{5}$ Given an arbitrary, fiberwise nondegenerate bilinear form $\omega$ on $T M, \omega^{\sharp}$ defines a bijection which may be lifted to the full tensor bundle over $M$ and which thus allows to identify different types (covariant, contravariant) of tensors ("lowering and raising of indices"). If we want $\Pi \in \Gamma(T M \otimes T M)$ to be the same object as $\omega \in \Gamma\left(T^{*} M \otimes T^{*} M\right)$ in this identification, $\Pi=\left(\left(\omega^{\sharp}\right)^{-1} \otimes\left(\omega^{\sharp}\right)^{-1}\right)(\omega)$, we find $\Pi^{\sharp}=\left(\omega^{\sharp}\right)^{-1, T}$. (Pseudo-)Riemannian geometry, where the bilinear form $\omega \equiv g$ is symmetric, is special in two ways: The transpose is irrelevant, $g^{i j}$ is the inverse to $g_{i j}$, and, maybe more important, symmetry of the bilinear form is the only case where index transport commutes with contraction of tensors. In the antisymmetric case considered here, we thus find the additional minus sign in the definition of $\Pi^{i j}$; moreover, now e.g. $A_{i} B^{i}=-A^{i} B_{i}$. (See also Appendix B of [26] for a discussion). 
Proposition 1 For a constrained system $C \hookrightarrow(M, \omega)$ the statements in part (i) and (ii), respectively, are equivalent to one another:

(i) (a) $C$ is first class.

(b) $(T C)^{\perp} \subset T C$.

(c) $\left.\Pi\right|_{\operatorname{Ann}_{M}(T C)}=0$, i.e. $\Pi(\alpha, \beta)=0$ for all $\alpha, \beta \in \operatorname{Ann}_{M}(T C)$.

(d) The embedding $\iota: C \rightarrow M$ is coisotropic, i.e. $(T C)^{\perp}$ is isotropic $\left(\left.\omega\right|_{(T C) \perp}=0\right)$.

(ii) (a) $C$ is second class.

(b) $(T C)^{\perp} \cap T C=0$.

(c) $\left.\Pi\right|_{\mathrm{Ann}_{M}(T C)}$ is nondegenerate.

(d) $\left.\omega\right|_{(T C)^{\perp}}$ is nondegenerate.

(e) $C$ is symplectic, i.e. $\iota^{*} \omega\left(\right.$ or $\left.\left.\omega\right|_{T C}\right)$ is nondegenerate.

In (ii), (b) 0 denotes the zero section.

Proof: Equivalence of (a) and (b) in (i) and (ii) follows immediately from Definition 1 with the preceding considerations. Likewise for (c) and (d), if we note that $\omega\left(v^{\alpha}, v^{\beta}\right)=$ $\Pi\left(\mathrm{d} \Phi^{\alpha}, \mathrm{d} \Phi^{\beta}\right)$; since, moreover, the righthand side of this equation is equal to $\left.\left\{\Phi^{\alpha}, \Phi^{\beta}\right\}\right|_{C}$, (c) in (i) and (ii) is recognized as the original definition of Dirac, formulated, however, without the use of constraint functions $\Phi^{\alpha}$ used to specify the embedded constraint surface C.

Concerning (i) it is now sufficient to establish equivalence between (b) and (d): $(T C)^{\perp} \subset$ $T C$ by definition implies isotropy of $(T C)^{\perp}$, and isotropy of $(T C)^{\perp}$ yields

$$
\operatorname{Ann}_{M}(T C)\left((T C)^{\perp}\right)=\left[\omega^{\sharp}\left((T C)^{\perp}\right)\right]\left((T C)^{\perp}\right)=0 .
$$

Concerning (ii) we next show (b) $\Leftrightarrow(\mathrm{e})$ : Let us assume that $\iota^{*} \omega$ is degenerate, which implies that there is a point $p \in C$ which has a nonzero tangent vector $v \in T_{p} C$ such that $\iota^{*} \omega(v, w)=0$ for all $w \in T_{p} C$. This is in contradiction to $T C \cap(T C)^{\perp}=0$. Likewise, if there is an isotropic tangent vector, i.e. a nonzero vector which is contained in both $T C$ and $(T C)^{\perp}, \iota^{*} \omega$ is obviously degenerate.

Finally, equivalence between (b) and (d) in (ii) may be shown likewise by noting that $T C=\left((T C)^{\perp}\right)^{\perp}$, so that we may replace $T C$ by $(T C)^{\perp}$ in the preceding argument.

Since we are interested in both presymplectic and Poisson manifolds as two different generalizations of symplectic manifolds, let us in the following suggest a generalization of the notions of first class and second class submanifolds to these cases, ensuring agreement on their symplectic intersection. In this context we want to keep the following characteristics of first and second class constraint surfaces $C$ : It should not be possible that $C$ is simultaneously first and second class (Dirac's classification is not exhaustive, but at least it should remain exclusive). Moreover, a second class constraint surface is a (nondegenerate) phase space of its own ( $C$ carries a natural symplectic structure), and a first class 
constraint surface is a phase space after factoring out gauge transformations which are generated by the flow of the constraints (in both cases, first and second class, this defines the reduced phase space). 1 On the other hand, at least for Poisson manifolds there already exists a reasonable notion of coisotropic submanifolds (cf., e.g., [3]). In this way we arrive at the following definition:

\section{Definition 2}

(i) Let $C$ be a (closed) submanifold of a presymplectic manifold $(M, \omega)$.

(a) $C$ is called coisotropic, if $(T C)^{\perp}$-as defined in (1) -is isotropic,

(b) it is called first class if $(T C)^{\perp} \subset T C$, and

(c) it is called second class, if $T C \cap(T C)^{\perp}=0$.

(ii) A (closed) submanifold $C$ of a Poisson manifold $(M, \Pi)$ is called coisotropic, if $\mathrm{Ann}_{M}(T C)$ is isotropic, i.e. $\left.\Pi\right|_{\mathrm{Ann}_{M}(T C)}=0$.

(iii) Let $C$ be a (closed) submanifold of a Poisson manifold $(M, \Pi)$ such that $\iota_{*} T C \subset$ $\Pi^{\sharp}\left(T^{*} M\right)$.

(a) $C$ is called first class if $0 \neq\left.\Pi^{\sharp} \mathrm{Ann}_{M}(T C)\right|_{x} \subset T_{x} C$ for any point $x \in C$, and

(b) second class if $\Pi^{\sharp} \operatorname{Ann}_{M}(T C) \cap T C=0$.

Using the fact that in the symplectic case $\Pi^{\sharp}$ defines an isomorphism between $\operatorname{Ann}_{M}(T C)$ and $(T C)^{\perp}$, it is easy to see that the respective definitions for presymplectic and Poisson manifolds coincide if $M$ is symplectic. In particular, the additional condition in part (iii) is always true in this case due to $\Pi^{\sharp}\left(T^{*} M\right)=T M$.

Remark: Conditions (a) and (b) in part (i) are not equivalent to one another if $(M, \omega)$ is not symplectic. In particular, there may be coisotropic second class submanifolds (iff $C$ is a symplectic submanifold of $M$ with $\left.(T C)^{\perp}=\operatorname{ker} \omega\right)$. On the other hand, in the Poisson case $\left.\Pi\right|_{\mathrm{Ann}_{M}(T C)}=0$ is equivalent to $\Pi^{\sharp} \operatorname{Ann}_{M}(T C) \subset T C$. Nonetheless, still a coisotropic submanifold of a Poisson manifold is not necessarily first class because the additional condition in part (iii) may be violated. In both cases, Poisson and presymplectic, a first class submanifold $C$ is coisotropic, but in general not vice versa.

It follows as in previous considerations that a first class submanifold of a presymplectic manifold $(M, \omega)$, with the pulled back 2 -form $\omega_{C}=\iota^{*} \omega$, is degenerate, i.e. the kernel has to be factored out to arrive at a reduced phase space, whereas a second class submanifold is always symplectic.

\footnotetext{
${ }^{6}$ For presymplectic manifolds the flow generated by a constraint is not uniquely defined. However, the ambiguity is the kernel of $\omega \in \Omega^{2}(M)$, which we thus want to include into the generators of the 'flow of gauge transformations'. This is achieved in the definition below.
} 
If we have a submanifold $C$ of a Poisson manifold $\left(M, \Pi_{M}\right)$, on the other hand, it is in general not possible to even define a Poisson or presymplectic structure on $C$ directly since we cannot use the pull back as in the (pre)symplectic case. However, one of the instances where this is possible occurs, if the annihilator is a Poisson ideal:

$$
\Pi_{M}^{\sharp}\left(\operatorname{Ann}_{M}(T C)\right)=0,
$$

i.e. $\Pi_{M}(\alpha, \cdot)=0$ for all $\alpha \in \operatorname{Ann}_{M}(T C)$. Using the isomorphism

$$
i:\left.T^{*} C \rightarrow T^{*} M\right|_{C} / \operatorname{Ann}_{M}(T C), \alpha \mapsto\left[\alpha_{M}\right]=\alpha_{M}+\operatorname{Ann}_{M}(T C)
$$

where $\alpha_{M}$ is some element of $\left.T^{*} M\right|_{C}$ fulfilling $\alpha_{M}(v)=\alpha(v)$ for all $v \in T C \subset T M$,

$$
\Pi_{C}(\alpha, \beta):=\left.\Pi_{M}\left(\left[\alpha_{M}\right],\left[\beta_{M}\right]\right)\right|_{C}
$$

leads to a well-defined Poisson tensor $\Pi_{C}$ on $C$, if (3) is satisfied.

Note that the condition (3) is stronger than the one required for coisotropic submanifolds, which only requires $\Pi^{\sharp} \operatorname{Ann}_{M}(T C) \subset T C$; correspondingly a general coisotropic submanifold of a Poisson manifold does not carry a canonical Poisson (or presymplectic) structure. The (coisotropic) submanifolds $C$ of smallest possible dimension fulfilling (3) are the symplectic leaves; in this case $\operatorname{Ann}_{M}(T C)=$ ker $\Pi^{\sharp}$ and $\Pi_{C}$ becomes nondegenerate. The symplectic form $\omega_{C}$ on the leaf $C$ is then defined by $\left(\omega_{C}\right)^{\sharp}=\left(\left(\Pi_{C}\right)^{\sharp}\right)^{-1}$.

The additional condition $\iota_{*} T C \subset \Pi^{\sharp}\left(T^{*} M\right)$ in part (iii) of Definition 2 ensures that $C$ is a subset of a symplectic leaf in $(M, \Pi)$. It is then always possible to define a symplectic structure on a first class or second class submanifold $C$ by pulling back the symplectic structure on the leaf to $C$. In this way, a first class submanifold acquires a degenerate presymplectic structure, whereas a second class submanifold always is symplectic.

In the following section we focus on closed constraint algebras, i.e. on a constrained system $\left(M, \omega,\left\{\Phi^{\alpha}\right\}\right)$ in which the constraints $\Phi^{\alpha}=0$ generate a Poisson subalgebra. This implies that

$$
\left\{\Phi^{\alpha}, \Phi^{\beta}\right\}_{M}(x)=\Pi_{P}^{\alpha \beta}(\Phi(x))
$$

holds for some $\Pi_{P}^{\alpha \beta}(\Phi)$. In the context of (5) the constraint surface $C$ is of first class, iff $\Pi_{P}(0)=0$ and of second class, iff $\operatorname{det} \Pi(0) \neq 0$.

For simplicity we will take $M$ to be connected in the following and require that the constraint functions $\Phi^{\alpha}$ can be used as part of a local coordinate system around any point in $M$ (instead of just points in $C \subset M$, which follows from the regularity and irreducibility of the constraints required in the beginning of this section).

\section{Poisson geometry from closed constraint algebras}

In this section we present results on the geometry of closed constraint algebras and the relation to the reduced phase space. This then yields an alternative procedure for a constraint reduction. 


\subsection{Symplectic dual pairs and related morphisms}

Define $P$ as the subset of $\mathbb{R}^{d}$ with coordinates $\Phi^{\alpha}$ which is in the image of $M$ under the constraint map $\phi: M \rightarrow P \subset \mathbb{R}^{d}, x \mapsto \Phi^{\alpha}(x)$ and endow $P$ with the bivector $\Pi_{P}=$ $\frac{1}{2} \Pi_{P}^{\alpha \beta}(\Phi) \partial_{\alpha} \wedge \partial_{\beta}$ appearing in the closed constraint algebra (5). In this way we have

$$
\left(C, \omega_{C}\right) \stackrel{\iota}{\hookrightarrow}(M, \omega) \stackrel{\phi}{\longrightarrow}\left(P, \Pi_{P}\right)
$$

Both arrows in this diagram are morphisms. However, there are two different categories involved: $C$ endowed with $\omega_{C}=\iota^{*} \omega$ is a presymplectic manifold (as before $\iota$ denotes the embedding of the constraint surface $C$ into $M$ ); note that in the present case the dimension of the kernel of $\omega_{C}$ is necessarily constant on $C$, and in the following we will include this condition on the kernel of the 2-form in the definition of a presymplectic manifold unless stated otherwise. $\left(P, \Pi_{P}\right)$, on the other hand, is Poisson. Correspondingly, $\iota: C \rightarrow M$ is a morphism of presymplectic manifolds and $\phi: M \rightarrow P$ a morphism of Poisson manifolds, where, in the first instance, $(M, \omega)$ is regarded as a (nondegenerate) presymplectic manifold and, in the second case, as a (nondegenerate) Poisson manifold.

Definition 3 A morphism $f$ between two (pre)symplectic manifolds $\left(M_{1}, \omega_{1}\right)$ and $\left(M_{2}, \omega_{2}\right)$, $a$ (pre)symplectic map, is a map $f: M_{1} \rightarrow M_{2}$ such that $f^{*} \omega_{2}=\omega_{1}$. A morphism $g$ between two Poisson manifolds $\left(M_{1}, \Pi_{1}\right)$ and $\left(M_{2}, \Pi_{2}\right)$, a Poisson map, is a map $g: M_{1} \rightarrow M_{2}$ such that $g_{*} \Pi_{1}=\Pi_{2}$.

An alternative characterization of a Poisson map makes use of the definition of coisotropy (Definition 2) and the notion of the graph $\Gamma_{g}=\left\{\left(m_{1}, g\left(m_{1}\right)\right): m_{1} \in M_{1}\right\} \subset M_{1} \times M_{2}$ of a $\operatorname{map} g: M_{1} \rightarrow M_{2}$ :

Lemma 1 ([27, 3]) $A$ map $g: M_{1} \rightarrow M_{2}$ between Poisson manifolds is a Poisson map if and only if its graph $\Gamma_{g}$ is coisotropic in $M_{1} \times \bar{M}_{2}$ where $\bar{M}_{2}$ has the negative Poisson structure of $M_{2}$.

Equivalently a Poisson map $g$ may be characterized by $g^{*}\{F, G\}_{M_{2}}=\left\{g^{*} F, g^{*} G\right\}_{M_{1}}$ for all $F, G \in C^{\infty}\left(M_{2}\right)$. Thus by construction $\phi: M \rightarrow P$ is Poisson (cf. e.g. (5)) and found to provide a surjective, submersive symplectic realization of $P$ :

Definition 4 A symplectic realization of a Poisson manifold $P$ is a Poisson map $\phi: M \rightarrow$ $P$ where $M$ is a symplectic manifold.

For our mathematical considerations we will drop the condition that $P \subset \mathbb{R}^{d}$ with $d=\operatorname{dim} P$. We then have to single out a point $0 \in P$ in order to define the constraint surface $C=\phi^{-1}(0)$. As the following theorem shows, any Poisson manifold $P$ can be obtained in the above way:

\footnotetext{
${ }^{7}$ Warning: In general (and in particular always for (6) in the instance of symplectic $C$ and $P$, as we will verify explicitly in subsection 3.2 below) a Poisson map (a (pre)symplectic map) between two symplectic manifolds is not symplectic (Poisson).
} 
Theorem 1 (Karasev, Weinstein [12, 13]) Every Poisson manifold has a surjective, submersive symplectic realization.

In a sense dual to this observation is the following embedding theorem for a presymplectic manifold:

Theorem 2 (Gotay [14]) Every presymplectic manifold $(M, \omega)$ can be coisotropically embedded into a symplectic manifold. This extension of $M$ is unique up to a local symplectomorphism in a neighborhood of $M$.

Later in this paper (Sec. (1) we will address the question as to whether there is an analogous, rather than dual, result for Poisson manifolds, namely whether any Poisson manifold $P$ can be embedded into a symplectic manifold $M$ such that the embedding becomes symplectic upon restriction to any leaf of $P$.

We may also compare this with the embeddings considered in the previous section (cf. in particular Definition Q): The embedding $\iota$ of a second class submanifold $C$ into a Poisson manifold cannot be Poisson. Indeed in order to be able to define some $\Pi_{C} \subset \Gamma\left(\Lambda^{2} T C\right)$ such that $\iota$ is Poisson $\left(\iota_{*} \Pi_{C}=\Pi_{M}\right)$, it is necessary and sufficient that $\left.\Pi_{M}^{\sharp}\left(T^{*} M\right)\right|_{C} \subset T C$, which is easily recognized as the condition (3), and which obviously can be defined only for $\operatorname{dim} C \geq \operatorname{rank} \Pi_{M}^{\sharp}$ (while a for a second class submanifold always $\operatorname{dim} C \leq \operatorname{rank} \Pi_{M}^{\sharp}$, equality holding for $C$ being a symplectic leaf). Nevertheless $C$ inherits a natural (nondegenerate) Poisson structure as a submanifold of a symplectic leaf. It may be quite interesting to reconsider (and possibly generalize) the (embedding) maps discussed in Definition 1 and Sec. 6 within the more general and unifying framework of Dirac structures (cf. footnote 目).

In the context of constrained systems $(M, \omega)$ with a closed constraint algebra (5) there is, under appropriate regularity conditions, also another canonical Poisson manifold $Q$ associated to it: Define an equivalence relation on $M$ by calling two points equivalent, if they lie on the same orbit generated by the Hamiltonian vector fields $v^{\alpha}=\left\{\cdot, \Phi^{\alpha}\right\}$ of the constraints. Assume that $Q:=M / \sim$ is a differentiable manifold (for a case where this condition is violated cf. e.g. Example 2 below) and denote the respective projection map from $M$ to $Q$ by $\pi$. Then $Q$ may be equipped with a Poisson bracket by $\pi^{*}\{f, g\}_{Q}:=$ $\left\{\pi^{*} f, \pi^{*} g\right\}_{M}$ for all $f, g \in C^{\infty}(Q)$. This indeed provides a well-defined bracket on $Q$ since due to the Jacobi identity for the Poisson bracket on $M$ the right-hand side is a function in the kernel of all the $v^{\alpha}$ and thus it can be written as the pull back of a function on $Q$. With this choice for the bracket, $\left(Q, \Pi_{Q}\right)$ is a Poisson quotient of $M$, i.e. the projection map $\pi: M \rightarrow Q$ is Poisson.

The two manifolds $Q$ and $P$ are not unrelated certainly. In fact we have

Proposition 2 With the assumptions above the two Poisson manifolds $Q$ and $P$ form a symplectic dual pair with respect to the original symplectic manifold $M$, in which the Poisson map $\pi: M \rightarrow Q$ is complete. 
Definition 5 ([3]) Two Poisson manifolds $\left(P, \Pi_{P}\right)$ and $\left(Q, \Pi_{Q}\right)$ form a symplectic dual pair with respect to a symplectic manifold $(M, \omega)$ if there are two Poisson maps $\phi:(M, \omega) \rightarrow$ $\left(P, \Pi_{P}\right), \pi:(M, \omega) \rightarrow\left(Q, \Pi_{Q}\right)$ with symplectically orthogonal fibers.

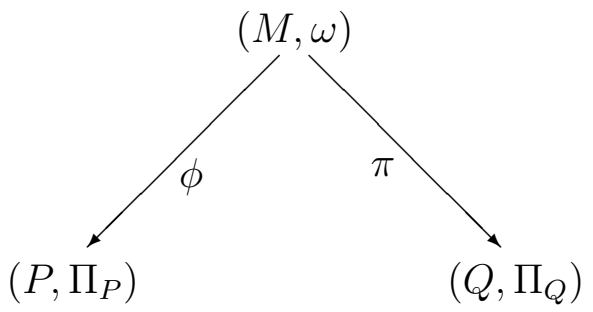

Definition 6 A Poisson map $\pi: M \rightarrow Q$ is complete, if the Hamiltonian vector field $X_{\pi^{*} h}=\left\{\cdot, \pi^{*} h\right\}$ generated by $\pi^{*} h$ in $M$ is complete for any function $h$ of compact support on $Q$.

Proof: To prove the first part of the above Proposition it is only necessary to note that the fibers are symplectically orthogonal iff the pull back of any function on $P$ (i.e. a function of the constraints) Poisson commutes with the pull back of any function on $Q=M / \sim$, i.e. a function $f$ satisfying $v^{\alpha} f=0$ for all $1 \leq \alpha \leq d$. Any function $g \in \phi^{*} C^{\infty}(P)$ can be expressed as a function of the $\Phi^{\alpha}$ only, for which we have $\left\{\Phi^{\alpha}, f\right\}=-v^{\alpha} f=0$, proving $\{g, f\}=0$ for any $f \in \pi^{*} C^{\infty}(Q), g \in \phi^{*} C^{\infty}(P)$ and so symplectic orthogonality of the $\pi$ and $\phi$-fibers.

The completeness of the projection map $\pi$ may be shown by adapting the proof of Proposition 6.6 in [3]: Let $h$ be a function of compact support on $Q$ which implies that $X_{h}=\{\cdot, h\}$ has a complete integral curve through some given point $q \in Q$. We now assume that the integral curve of $X_{\pi^{*} h}$ through any point $m \in \pi^{-1}(q)$ is not complete, i.e. there is (without loss of generality) a maximal parameter $t_{+}$beyond which the curve cannot be defined. Through the projection to $Q$ by $\pi$ of the final point $m_{+}$belonging to $t_{+}$, there is, however, an extension of the integral curve which can be lifted to $M$ to a curve through some point $m^{\prime} \in \pi^{-1}\left(\pi\left(m_{+}\right)\right.$) (not necessarily identical to $m_{+}$). Using the action generated by the constraints $\Phi^{\alpha}$ on $M$, by which we defined the equivalence relation $\sim$, we can construct local symplectomorphisms $s$ from a neighborhood of $m^{\prime}$ to a neighborhood of $m_{+}$such that $s\left(m^{\prime}\right)=m_{+}$(e.g., by following integral curves of the $v^{\alpha}$ in a chosen ordering, where the parameter lengths of all the integral curves are determined so as to fulfill $s\left(m^{\prime}\right)=m_{+}$and are the same for all points in the neighborhood of $\left.m^{\prime}\right)$. Since $X_{\pi^{*} h}$ is by definition invariant under the flow, the local symplectomorphism transports a piece of its integral curve through $m^{\prime}$ to a piece of the integral curve through $m_{+}$. This is a contradiction to our assumption that the original integral curve through $m$ is inextendible beyond $m_{+}$which proofs completeness of the map $\pi$. 
The orthogonality in the above proof could have been infered also from the results of Sec. 2, where the span of the vector fields $v^{\alpha}$ generating the orbits (the fibers of the map $\pi$ ) was identified with $(T C)^{\perp}$, the symplectically orthogonal to $T C$, which in turn is the tangent bundle to a (generalized) "constraint surface" $C$ defined by the preimage of some point in $P$ (the fibers of the map $\phi$ ). Note in this context that orthogonality of the fibers does not imply that they are transversal to one another (this is only the case for second class constraints). In particular, for first class constraints the fibers of $\pi$ are even submanifolds of the fibers of $\phi$.

In the rest of this section we want to clarify the relation between the factor space $Q$ and the reduced phase space $R$. For instance if $P$ is symplectic, so that the constraints $\Phi^{\alpha}$ are second class, $R$ coincides just with $\left(C, \omega_{C}\right)$ in $(\overline{6})$. We then will show that, under some rather mild conditions specified below, $R$ and $Q$ are symplectomorphic. (Note that $P$ symplectic implies $Q$ symplectic because their pre-images in the symplectic manifold $M$ are symplectically orthogonal due to Prop. 2.) Thus in this case we can trade in the standard procedure of reducing the corresponding second class constrained system on $M$ by restriction to the constraint surface $C$ for taking the factor space of $M$ with respect to the flow generated by the second class constraints. This second approach is in its spirit more closely related to the (in physical systems generically better understood) reduction procedure used for constrained systems with purely first class constraints. In fact, as we will show, $M$ may be regarded as a constraint surface in a higher dimensional (extended) symplectic manifold $\widetilde{M}$ characterized by first class constraints, the orbits of which on $M \hookrightarrow \widetilde{M}$ coincide with the original orbits of $v^{\alpha}$ on $M$.

In the following we will first focus on this case of a symplectic target $\left(P, \Pi_{P}\right)$ (corresponding to second class constraints) before we then consider the general case.

\subsection{Second class constraints}

By assumption, $\left(P, \Pi_{P}\right)$ is symplectic (we denote the symplectic form inverse to $\Pi_{P}$ by $\Omega_{P}$ in the following). Then any Poisson map $\phi: M \rightarrow P$ is a submersion [3]. If in addition $\phi$ is complete, we have

Lemma 2 Let $\phi$ be a complete Poisson map from a Poisson manifold $M$ to a symplectic manifold $\left(P, \Omega_{P}\right)$. Then $M$ is a fiber bundle over $P$ with projection map $\phi$ which is endowed with a natural flat connection.

The proof [3] proceeds by showing that any vector $v \in T P$ can be lifted horizontally to a vector in $T M$ by pulling back the covector $v\lrcorner \Omega_{P}$. In this way one obtains a connection which is flat since the Lie bracket of two horizontal vector fields is again horizontal. To see this, one uses canonical coordinates on $P$ and completeness of the map $\phi$.

In our situation of regular second class constraints, we have

Theorem 3 Let $\Phi^{\alpha}, 1 \leq \alpha \leq d$ be regular constraint functions with nonempty constraint surface $C=\phi^{-1}(0)$ on a connected symplectic manifold $(M, \omega)$ with a complete Poisson map $\phi:(M, \omega) \rightarrow\left(P, \Pi_{P}\right), x \mapsto \Phi^{\alpha}(x)$ such that the Poisson manifold $\left(P, \Pi_{P}\right)$ is symplectic. 
If $Q=M / \sim$, which is symplectically dual to $\left(P, \Pi_{P}\right)$ with respect to $(M, \omega)$, is a manifold, then it is covered by the constraint surface $\left(C, \omega_{C}\right)$. This is always the case if the holonomy group of the flat connection of Lemma 0 is finite; if it is trivial, the covering by $C$ is a symplectomorphism.

Proof: By construction $C$, defined by $\Phi^{\alpha}=0$ for all $\alpha$, is embedded as a submanifold into $M, \iota: C \rightarrow M$, and $\omega_{C}=\iota^{*} \omega$. Now define an equivalence relation for points on $C$ by calling two points on $C$ equivalent if they are connected by a constraint orbit in $M$. Taking the corresponding factor space yields $Q$ as a topological space. Here it is essential that any orbit $\mathcal{O}_{p}$ of the Hamiltonian vector fields generated by $\Phi^{\alpha}$ through a point $p \in M$ intersects the constraint surface $C$ at least once.

This is indeed the case due to completeness of the map $\phi$ : if $p \notin C$, the point $p$ is mapped to $0 \neq \phi(p) \in P$, which can be connected to $0 \in P$ along trajectories of Hamiltonian vector fields, chosen to be of compact support, in $P$ since $P$ is symplectic. These trajectories can be carried to $M$ by pulling back their Hamiltonian functions with $\phi$. Due to the completeness of $\phi$, they can be extended to arbitrary parameters such that we reach a point in the pre-image of $0 \in P$. This point lies on the constraint surface $C$.

In general, there will be more than one intersection point. Thanks to Lemma 2 the bundle $\phi: M \rightarrow P$ with typical fiber $C=\phi^{-1}(0)$ comes equipped with a flat connection whose holonomy provides an action of the fundamental group $\pi_{1}(P)$ on $C$. The set of all points in the intersection of $C$ with an orbit $\mathcal{O}$ through a point $p \in C$ is given by the orbit through $p$ of the action of $\pi_{1}(P)$. The number of copies of $p$ obtained in such a way is given by the number $k$ (maybe infinite) of elements in the factor space $\pi_{1}(P) / F_{p}$, where $F_{p}$ is the isotropy subgroup of $\pi_{1}(P)$ in $p$. Since $\pi_{1}(P)$ is discrete, this number is constant on any connected component of $C$. Factoring $C$ with the action of $\pi_{1}(P)$ yields a $k$-fold covering $C \rightarrow Q$, where $Q$ is obtained by the projection map $\pi: M \rightarrow Q$ along the orbits $\mathcal{O}$ as in Proposition 2. If $k$ is finite, the action of $\pi_{1}(P)$ is properly discontinuous and $Q$ is a manifold (see Example 2 for a case with an infinite holonomy group). If $k=0$ (trivial holonomy), $C \cong Q$ topologically.

We next show that $\omega_{C}$ factors through to $Q$ (under the assumption that $Q$ is a manifold). Hamiltonian vector fields generated by $\Phi^{\alpha}$ yield a symplectic map between neighborhoods of the surfaces $\phi(x)=\phi_{0}$ and $\phi(x)=\phi_{1}$ for any constants $\phi_{0}$ and $\phi_{1}$. This also holds true if $\phi_{0}=\phi_{1}=0$, in which case we have the map identifying points on $C$ to result in $Q$ used above. Consequently, the symplectic structure $\omega_{C}$ on $C$ can be projected down to yield the symplectic manifold $\left(Q, \Omega_{Q}\right)$.

It remains to show that the map $\pi:(M, \omega) \rightarrow\left(Q, \Omega_{Q}\right)$ is indeed Poisson. (The rest, such as symplectic orthogonality of the $\pi$ - and $\phi$-fibers, follows from (or as in the proof of) Proposition 2.) For this purpose we introduce some further notation: Decompose the tangent and cotangent space at $x \in C \subset M$ according to the splitting induced by $C$ and the orbit $\mathcal{O}_{x}$. More explicitly, $T_{x} M \cong T_{x} C \oplus T_{x} \mathcal{O}$ and $T_{x}^{*} M=\operatorname{Ann}_{M}\left(T_{x} \mathcal{O}\right) \oplus \operatorname{Ann}_{M}\left(T_{x} C\right) \cong$ $T_{x}^{*} C \oplus T_{x}^{*} \mathcal{O}$, where $\cong$ denotes canonical isomorphisms, used to identify respective vector spaces. Denote by $\iota_{i}$ and $p_{i}, i=1,2$, the respective embeddings and projections to the first

${ }^{8}$ We could have replaced $T_{x} \mathcal{O}$ by $\left(T_{x} C\right)^{\perp}$, cf. Sec. 2. 
and second factor in $T_{x} M$; thus e.g. $\iota_{1}: T_{x} C \rightarrow T_{x} M$. Then $\pi_{i}:=\iota_{i} \circ p_{i}$ is the corresponding projection operator inside $T_{x} M$. Proceed likewise for $T_{x}^{*} M$ with bars on the respective maps; thus, e.g., $\bar{\iota}_{1}: T_{x}^{*} C \rightarrow T_{x}^{*} M$ etc.

In this notation, the condition defining $\omega_{C}, \omega_{C}=\iota^{*} \omega$, takes the form $\omega_{C}^{\sharp}=\bar{p}_{1} \omega^{\sharp} \iota_{1}$, while orthogonality of the fibers becomes $p_{2} \Pi^{\sharp} \bar{\pi}_{1}=0=p_{1} \Pi^{\sharp} \bar{\pi}_{2}$. From this we need to prove that $\pi$ as defined above is Poisson, i.e. that $p_{1} \Pi^{\sharp} \bar{\iota}_{1}=\Pi_{C}^{\sharp}$. This indeed follows since

$$
p_{1} \Pi^{\sharp} \bar{\iota}_{1} \omega_{C}^{\sharp}=p_{1} \Pi^{\sharp} \bar{\iota}_{1} \bar{p}_{1} \omega^{\sharp} \iota_{1}=p_{1} \Pi^{\sharp}\left(\bar{\pi}_{1}+\bar{\pi}_{2}\right) \omega^{\sharp} \iota_{1}=-\mathbb{I}_{T_{x} C}
$$

Example 1: (generalized from [7]) Let $M:=\mathbb{R}^{2} \backslash\{(0,0)\}$ be the punctured plane with canonically conjugate coordinates $(q, p)$ and define on $M$ the constraints

$$
\Phi_{n}^{1}:=\sqrt{q^{2}+p^{2}} T_{n}\left(q\left(q^{2}+p^{2}\right)^{-\frac{1}{2}}\right)-1 \quad, \quad \Phi_{n}^{2}:=p U_{n-1}\left(q\left(q^{2}+p^{2}\right)^{-\frac{1}{2}}\right)
$$

for a fixed $n \in \mathbb{N}$ using the Chebyshev polynomials $T_{n}$ and $U_{n}$ of the first and second kind. With $T_{n}(\cos \theta)=\cos n \theta$ and $U_{n}(\cos \theta)=\sin [(n+1) \theta] / \sin \theta$, one can see that $\left\{\Phi_{n}^{1}, \Phi_{n}^{2}\right\}=n$, and thus $P=\phi(M)=\mathbb{R}^{2} \backslash\{(-1,0)\}$ is a symplectic manifold.

In polar coordinates defined by $q=r \cos \phi, p=r \sin \phi$ it follows easily that the constraint surface $C=\{(\cos (k \pi / n), \sin (k \pi / n)): 0 \leq k \leq n\}$ consists of $n$ points which are all connected by the single orbit generated by the constraints in $M$. The map $C \rightarrow Q$ is an $n$-fold covering.

Example 2: Let $M:=T^{2} \times \mathbb{R}^{2} \ni\left(q_{1}, p_{1} ; q_{2}, p_{2}\right)$ with constraints $\Phi^{1}:=q_{1}+\omega p_{1}+q_{2}$ and $\Phi^{2}:=p_{2}$ (the first constraint function is taken modulo 1 such that $P=S^{1} \times \mathbb{R}$ ).

If $\omega=0$, the constraint surface is homeomorphic to $S^{1} \times \mathbb{R}$ where the $S^{1}$-factor comes from the unaffected coordinate $p_{1}$, and the $\mathbb{R}$-factor is the regular curve (a helix) $q_{1}=-q_{2}$ (mod 1) lying on the cylinder $S^{1} \times \mathbb{R} \subset M$ with coordinates $q_{1}, q_{2}$. The flow generated by the second constraint intersects the constraint surface of the first constraint in an infinite number of points of the form $\left(q_{1}, p_{1},-q_{1}+m, 0\right)$ for $m \in \mathbb{Z}$, and vice versa. Since the corresponding identifications form a discrete set of translations in the $q_{2}$-direction of constant shift, the factor space $Q$ is a manifold.

The situation is unchanged if $\omega$ is nonvanishing but rational. But if $\omega$ is irrational, the projection of the constraint surface to the cylinder appearing above will be dense. There are still infinitely many intersection points, and this time the factor space is not a differentiable manifold. This also follows from the fact that the orbits generated by the constraints are dense in a suitable subset of $M$, and so $Q=M / \sim$ cannot be a manifold.

Theorem 3 can be used to define a natural presymplectic form on $M$ :

Corollary 1 ( [7]) Under the assumptions of Theorem 3 the symplectic manifold $(M, \omega)$ carries a presymplectic form

$$
\bar{\omega}:=\omega-\phi^{*} \Omega_{P}
$$

whose kernel is tangent to the orbits generated by the $\Phi^{\alpha}$. 
Proof: By definition $\bar{\omega}$ is closed. From Theorem [3, in particular the fact that the fibers of the dual pair are symplectically orthogonal, it follows that $\bar{\omega}$ vanishes when applied to vector fields tangential to the orbits $\mathcal{O}$. This can also be seen directly: By definition, the Hamiltonian vector field $X_{\Phi^{\alpha}}$ generated by $\Phi^{\alpha}$ satisfies $\left.X_{\Phi^{\alpha}}\right\lrcorner \omega=\mathrm{d} \Phi^{\alpha}$. Furthermore, by definition of $\Omega_{P}$, we have

$$
\left.X_{\Phi^{\alpha}}\right\lrcorner \phi^{*} \Omega_{P}=\left(\Omega_{P}\right)_{\beta \gamma} X_{\Phi^{\alpha}}\left(\Phi^{\beta}\right) \mathrm{d} \Phi^{\gamma}=-\left(\Pi_{P}^{-1}\right)_{\beta \gamma} \Pi_{P}^{\beta \alpha} \mathrm{d} \Phi^{\gamma}=\mathrm{d} \Phi^{\alpha}
$$

so that $\left.X_{\Phi^{\alpha}}\right\lrcorner \bar{\omega}=0$.

It is clear from the proof of Theorem 3 that we have a trivial covering by $C$ if $\pi_{1}(P)=0$ :

Corollary 2 If $P$ is simply connected, then $\left(P, \Pi_{P}\right)$ and $\left(C, \omega_{C}\right)$ form a symplectic dual pair with respect to $(M, \omega)$.

This is always the case if $M$ is simply connected, as we will now demonstrate. In Theorem 3 and its proof we made use of the fact that $M$ may be considered as a fiber bundle over $P$ with typical fiber $C=\phi^{-1}(0)$, the constraint map $\phi$ playing the role of the projection from $M$ to $P$. However, if $Q$ is a manifold, $\left(Q, \Pi_{Q}\right)$ is symplectic and, according to Proposition 2, the respective projection map $\pi$ is a complete Poisson map. We thus may apply Lemma 2 also to this context and conclude as in the proof of Theorem 3 that the orbit $\left(\mathcal{O}, \omega_{\mathcal{O}}\right)$ (with symplectic form $\omega_{\mathcal{O}}$ induced on it by $\omega$ ) covers $\left(P, \Omega_{P}\right)$. Moreover, this covering map is a symplectomorphism if the holonomy of the respective flat connection is trivial, which in particular is always the case if $\pi_{1}(Q)=0$.

Proposition 3 If $M$ is simply connected (and connected), also $P$ and $C$ are simply connected and connected.

Proof: For the fiber bundle $\mathcal{O} \hookrightarrow M \rightarrow Q$ we have the exact homotopy sequence (cf. e.g. 28])

$$
\cdots \rightarrow \pi_{1}(M) \rightarrow \pi_{1}(Q) \rightarrow \pi_{0}(\mathcal{O}) \rightarrow \cdots
$$

Since by assumption $\pi_{1}(M)=0$ and by definition $\pi_{0}(\mathcal{O})=0$, it follows that $\pi_{1}(Q)$ vanishes. According to the considerations above, this implies that the fiber bundle $\mathcal{O} \hookrightarrow M \rightarrow Q$ is trivial and that its fiber is isomorphic to $P$ (here we use the holonomy of Lemma 2 for which the completeness of the map $\phi: M \rightarrow P$ is important); thus $M \cong Q \times P$. This in turn yields $\pi_{1}(M)=\pi_{1}(Q) \oplus \pi_{1}(P)$, resulting in $\pi_{1}(P)=0$. Thus $Q \cong C, \pi_{1}(C)=0$, and with $M$ being connected, finally also $P$ and $Q$ (or $C$ ) are connected.

This result can be helpful because $M$ is the original symplectic manifold and may thus be easier accessible than $P$ in particular cases. We also note that $P$ being simply connected is only a sufficient condition for $Q \cong C$. The essential object which determines $Q$ is the holonomy of the flat connection of Lemma 2 , which certainly can be trivial even if $P$ is not simply connected. In other words, it is the behavior of the $v^{\alpha}$-orbits in $M$ which determines 
whether the reduced phase space $C$ itself and $P$ are symplectically dual with respect to $M$ (and consequently then $C$ may be obtained as factor space by projection $\pi$ along the orbits).

Example 3: Let $M=T^{*}\left(S^{1} \times \mathbb{R}\right)$ with canonically conjugate coordinates $\left(x, y ; p_{x}, p_{y}\right)$, where $x \equiv x+1$, and with constraints $\Phi^{1}:=x, \Phi^{2}:=p_{x}$. Then $P=T^{*} S^{1}$ is not simply connected, but still $R \equiv C=Q=T^{*} \mathbb{R}$.

According to Proposition 3, $M$ being simply connected implies $Q \cong C$ and also that $C$ is connected. In Example 1, $M$ was not simply connected, $C$ was not connected and $C$ was a nontrivial covering of $Q$, i.e. we had Gribov copies (a Gribov copy of a point on the constraint surface is a different point on the constraint surface which lies on the same orbit generated by the constraints [7, 29]). As the following example demonstrates, this can also happen if $C$ is connected (but $M$ still is not simply connected):

Example 4: Let $M$ be the 4-torus with canonically conjugate coordinates $\left(x, p_{x} ; y, p_{y}\right) \in$ $T^{2} \times T^{2}$ which are identified modulo 1 . The constraints are $\Phi^{1}:=n x+y$ and $\Phi^{2}:=p_{x}$ with $1<n \in \mathbb{N}$ such that $\left\{\Phi^{1}, \Phi^{2}\right\}=n$ and the constraint surface $C \cong T^{2}$ characterized by $\left(x, 0,-n x, p_{y}\right)$ is connected.

The Hamiltonian vector fields generated by the constraints are $v^{1}=-n \mathrm{~d} / \mathrm{d} p_{x}-\mathrm{d} / \mathrm{d} p_{y}$ and $v^{2}=\mathrm{d} / \mathrm{d} x$. Their orbit $\mathcal{O}$ parameterized by $t_{1}, t_{2}$ through the point $\left(x_{0}, 0,-n x_{0}, p_{y, 0}\right)$ is

$$
\exp \left(t_{1} v^{1}+t_{2} v^{2}\right)\left(x_{0}, 0,-n x_{0}, p_{y, 0}\right)=\left(x_{0}+t_{2},-n t_{1},-n x_{0}, p_{y, 0}-t_{1}\right) .
$$

It intersects $C$ if and only if $t_{1}=m_{1} / n, t_{2}=m_{2} / n$ with $m_{1}, m_{2} \in \mathbb{Z}$. On the other hand, points on $\mathcal{O} \cong T^{2}$ coincide if $t_{1}$ or $t_{2}$ are changed by an integer. Therefore, the intersection $\mathcal{O} \cap C$ consists of $n^{2}$ points, which are obtained with $1 \leq m_{1}, m_{2} \leq n$.

Thus, $C$ is connected, $M$ not simply connected and we have $n^{2}$ Gribov copies. We note that the map $\left.\phi\right|_{\mathcal{O}}:\left(x_{0}+t_{2},-n t_{1},-n x_{0}, p_{y, 0}-t_{1}\right) \mapsto\left(n t_{2},-n t_{1}\right)$ also is an $n^{2}$-fold covering of $P \cong T^{2}$.

So the connectedness of the constraint surface $C$ (which coincides with the reduced phase space $R$ of physical interest) alone is not sufficient for the absence of Gribov copies, i.e. to guarantee together with completeness of $\phi$ that any orbit $\mathcal{O}$ generated by the constraints intersects $C$ in precisely one point. However, in the above example, $P$ is not a subset of $\mathbb{R}^{2}$, which would usually be the case in physical models (so, e.g., $\Phi^{1} \notin C^{\infty}(M) \equiv$ $C^{\infty}(M, \mathbb{R})$ and, correspondingly, the orbit generating vector fields $v^{1}$ and $v^{2}$ are only $l o-$ cally Hamiltonian). It would be interesting to clarify whether the condition $\pi_{0}(C)=0$ is sufficient if $P$ is a subset of $\mathbb{R}^{d}, d=\operatorname{dim} P$.

We now summarize our findings in a diagram: 


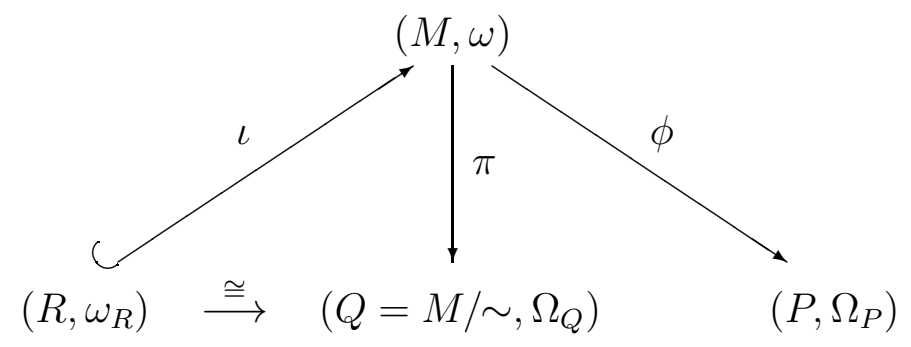

In the present subsection we considered the case of a symplectic target $\left(P, \Omega_{P}\right)$ (the general case of a Poisson manifold will be considered in the subsequent subsection). For simplicity we take the original unconstrained phase space $(M, \omega)$ to be connected. We now require the orbit space $Q=M / \sim$ to be a differentiable manifold (the orbits are generated by the constraints $\Phi^{\alpha}$ ). The right hand side of the diagram then follows from Proposition 1 , yielding $\pi$ to be a complete Poisson map. When $P$ is symplectic, the constraints are second class and the reduced phase space $\left(R, \omega_{R}\right)$ coincides with the constraint surface $\left(C, \omega_{C}\right)$ as embedded in $M$. Thus with (6) we obtain the embedding of $\left(R, \omega_{R}\right)$ into $M$ in the diagram. Requiring that the constraint map $\phi$ is complete, too, we showed in the present subsection that $\left(R, \omega_{R}\right)$ covers the Poisson quotient $Q$ via the map $\pi \circ \iota$. Moreover, if (as a sufficient but not necessary condition) $\pi_{1}(P)=0$ (which in turn follows e.g. if $M$ is simply connected), this covering is an isomorphism ('no Gribov copies'). (In [7] also another potential source for the map $R \rightarrow Q$ not to be an isomorphism was mentioned and backed up by an explicit example, namely systems where the map $\phi$ restricted to an orbit is not surjective. This qualitatively different case of a Gribov problem (corresponding to 'gauge orbits' not intersecting the 'gauge fixing surface' $C$ ) is excluded by the requirement that $\phi$ should be complete.)

In this favorable case of an isomorphism we thus obtain the reduced phase space $\left(R, \omega_{R}\right)$ not only by restriction to the constraint surface $C \subset M$ (standard procedure for reducing second class constraints), but instead may consider the factor space $Q$ of $M$ with respect to the orbits generated by the constraints. This is more closely related to the standard and generally more preferred treatment of first class constraints.

In fact, the analogy with first class constraints can be made even more precise: According to Corollary 1, $M$ may be equipped with a presymplectic form $\bar{\omega}$, the kernel of which coincides with the orbits to be factored out. Together with Corollary 2 we have

Corollary 2' If the Poisson map $\phi$ of the constraints is complete and the target $P$ symplectic and simply connected, there exists a symplectomorphism from $(M, \omega)$ to $\left(R, \omega_{R}\right) \times$ $\left(P, \Omega_{P}\right)$ with canonical projections to both factors. The reduced phase space may then be obtained by projecting to the first factor or, equivalently, by taking the factor space of $(M, \bar{\omega})$ with respect to the kernel of the presymplectic form $\bar{\omega}$ defined in (8).

The presymplectic manifold $(M, \bar{\omega})$ can then in turn be embedded coisotropically into some higher dimensional symplectic manifold $(\widetilde{M}, \widetilde{\omega})$. This extended phase space is equipped with first class constraints such that $M$ is the level zero set of the constraints and $\bar{\omega}$ the 
pull back of $\widetilde{\omega}$ with respect to the corresponding embedding map. In this way, $\left(R, \omega_{R}\right)$ can be obtained by standard reduction of the extended phase space $(\widetilde{M}, \widetilde{\omega})$ constrained by first class constraints. In section 3.4 we will provide two explicit possibilities for constructing such a constrained extended phase space $\widetilde{M}$, in one of which the constraints are even Poisson commuting (abelian). Within this reformulation, the above mentioned orbits of the (originally second class) constraints $\Phi^{\alpha}, \alpha=1, \ldots, d$, become just the standard gauge orbits of the extended system (generated by $d$ first class constraints) and the original constraint surface $C$ corresponds to one possible choice of a gauge (which exists globally due to the absence of a Gribov problem - now in the standard use of the terminology - as a consequence of the assumptions specified above).

Let us add a remark concerning the arrows in diagram (9): As remarked already before, they are morphisms of respective categories. So $\iota$ as well as the covering map $\iota \circ \pi$ are symplectic maps and $\pi, \phi$ are Poisson. Note that neither of the last two maps is symplectic, since $\pi^{*} \Omega_{Q}=\omega-\phi^{*} \Omega_{P} \neq \omega$. Similarly, $\iota$ is also not Poisson (except for $C=M$ ), since a Poisson map between symplectic manifolds is always a submersion [3]. Only the covering map is symplectic and Poisson. Likewise remarks apply to the diagrams below.

We finally remark that it is a direct consequence of Proposition 3 that, provided $M$ is connected and simply connected, so are $\left(R, \omega_{R}\right)$ and $\bar{P}:=\left(P,-\Pi_{P}\right) \cong\left(P,-\Omega_{P}\right)$. Moreover, in this case $M \cong R \times P$ and the maps $\pi$ and $\phi$, which then are just projections to the first and second factor, respectively, have constant rank. This demonstrates that $R$ and $\bar{P}$ are Morita equivalent (cf [3] for the definition) with respect to $(M, \omega)$.

\subsection{The general case}

Let us first consider the other extreme case opposite to the one of Sec. 3.2 and cast the standard reduction of a system of first class constraints into a diagram similar to the one at the end of the previous subsection. This yields the following (commutative) diagram:

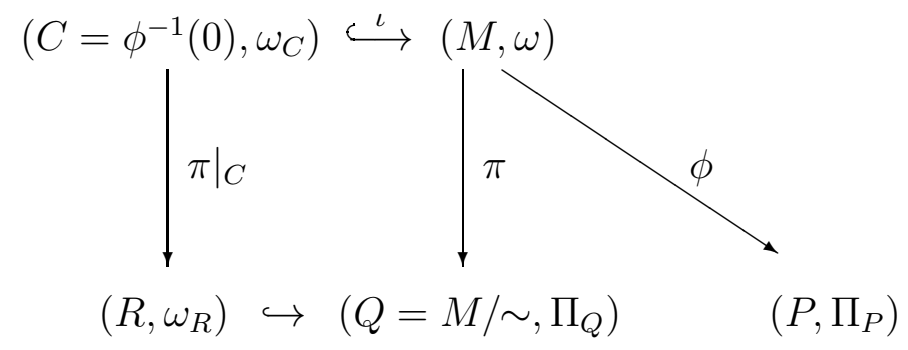

The right hand side is again taken from Prop. 2 (the assumption from there that $Q=M / \sim$ is a differentiable manifold is understood to hold here too). By definition the constraint surface $C$ is the preimage of zero with respect to the constraint map $\phi$. Restricting the projection map $\pi$ to $C$, which is equivalent to factoring out the flow generated by the kernel of $\omega_{C}$, yields the standard reduced phase space $R$. 
Proposition 4 Let $\left(R, \omega_{R}\right)$ be the reduced phase space of a first class constrained system with the assumptions of Prop. 6 . Then $R$ is a symplectic leaf in the orbit space $\left(Q=M / \sim, \Pi_{Q}\right)$ which is obtained by factoring out the constraint orbits.

Proof: Note first that there is a natural embedding of $R$ as a subset of $Q$, since the flow of the constraints does not leave $C$ being the preimage of a symplectic leaf (namely the origin) in $\left(P, \Pi_{P}\right)$.

To prove that the embedding is as a symplectic leaf, we first note that $\Pi_{Q}^{\sharp}\left(\operatorname{Ann}_{Q}(T R)\right)=$ 0 if $R$ is the reduced phase space. This follows from the fact that $\operatorname{Ann}_{Q}(T R)$ is spanned by total derivatives of the constraints which on $R$ Poisson commute with each other as well as with elements of $\left.T^{*} Q\right|_{R} / \operatorname{Ann}_{Q}(T R)$ since this space is spanned by total derivatives of physical observables which by definition commute on $R$ with the constraints. This shows that we can define a Poisson structure $\Pi_{R}$ on $R$ using (四):

$$
\{f, g\}_{R}=\Pi_{R}(\mathrm{~d} f, \mathrm{~d} g)=\left.\Pi_{Q}\left((\mathrm{~d} f)_{Q},(\mathrm{~d} g)_{Q}\right)\right|_{R}
$$

using the isomorphism $i:\left.T^{*} R \rightarrow T^{*} Q\right|_{R} / \operatorname{Ann}_{Q}(T R), \alpha \mapsto \alpha_{Q}+\operatorname{Ann}_{Q}(T R)$. If this Poisson structure is nondegenerate, $R$ is an open subset of a symplectic leaf in $Q$.

We proceed by showing that the pull back of $\{f, g\}_{R}$ under $\left.\pi\right|_{C}$ coincides with the bracket computed using the pull back of the symplectic structure on $M$. This will imply that $\Pi_{R}$ coincides with the structure of $R$ as the reduced phase space which by definition is nondegenerate.

In a first step we use the fact that $\pi$ is Poisson and obtain

$$
\left.\pi\right|_{C} ^{*}\{f, g\}_{R}=\left.\Pi_{M}\left(\pi^{*}(\mathrm{~d} f)_{Q}, \pi^{*}(\mathrm{~d} g)_{Q}\right)\right|_{C} .
$$

Here we need the image under the map $i$ of an exact 1-form $\mathrm{d} f$. More precisely, we want to show that $\alpha_{Q}$ can be chosen to be exact if $\alpha=\mathrm{d} f$ is exact. To construct a function $f_{Q}$ on $Q$ with $\alpha_{Q}=\mathrm{d} f_{Q}$, we choose a tubular neighborhood of $R$ in $Q$, cover it with open subsets in which we can use local coordinates of $R$ together with transversal coordinates, and use a partition of unity subordinate to these neighborhoods. First we can extend $f$ to a function $f_{\mathcal{U}}$ on any local neighborhood $\mathcal{U}$ by requiring that $f_{\mathcal{U}}$ does not depend on the transversal coordinates. Using the partition of unity, we arrive at a smooth function which is defined on the full tubular neighborhood of $R$ and which equals $f$ when pulled back to $R$. Multiplying this function with a smooth function which vanishes outside the tubular neighborhood and equals one on $R$ defines a smooth function $f_{Q}$ defined on $Q$. Because $\left.f_{Q}\right|_{R}=f$, we have $v\left(f_{Q}\right)=v(f)$ for any vector $v$ tangential to $R$ such that we can choose $(\mathrm{d} f)_{Q}=\mathrm{d} f_{Q}$ as representative in $\left.T^{*} Q\right|_{R} / \operatorname{Ann}_{Q}(T R)$. We use this relation to compute the pull back of the Poisson bracket

$$
\left.\pi\right|_{C} ^{*}\{f, g\}_{R}=\left.\Pi_{M}\left(\mathrm{~d} \pi^{*} f_{Q}, \mathrm{~d} \pi^{*} g_{Q}\right)\right|_{C}=\left.\left\{\pi^{*} f_{Q}, \pi^{*} g_{Q}\right\}\right|_{C} .
$$

Owing to the construction of $f_{Q}$ above, $\pi^{*} f_{Q}$ is a function on $M$ which is constant along constraint orbits and whose values on the orbits through $C$ coincide with $f$. This shows 
that the pull back to $C$ of the symplectic structure on $R$ as a leaf in $Q$ coincides with the symplectic structure of $M$ restricted to $C$.

Completeness of the map $\pi$ implies that $R$ is a symplectic leaf in $Q$, not just an open subset of a leaf: Assume that $R$ is contained in but not identical to a leaf $L$ of $Q$ and choose a point $r \notin R$ in the boundary of $R$ in $L$. We can choose a function $h$ on $Q$ which is supported only on some neighborhood of $r$ and which generates a Hamiltonian vector field $X_{h}$ tangential to $L$. Using the trajectories of $X_{h}$, we can connect the point $r \notin R$ to a point $r_{0} \in R$. Due to completeness, the pull back $\pi^{*} h$ generates a complete Hamiltonian vector field on $M$. Furthermore, it is tangential to $C$ because $X_{h}$ was chosen to be tangential to $L$. Thus, there are points in $\pi^{-1}(r)$ and $\pi^{-1}\left(r_{0}\right)$ which both lie in $C \subset M$ and so are projected to $R$ under $\pi$. Therefore, $r \in R$ contradicting our assumption $R \neq L$.

Thus for the reduction process in a system of first class constraints forming a closed constraint algebra we may exchange the order of restriction to a submanifold and taking the factor space. So, in this case, there are two equivalent, 'dual' perspectives of the reduction process, the one using presymplectic geometry in an intermediary step and the other one using Poisson geometry.

The case of a general Hamiltonian system with a closed constraint algebra is now basically a combination of the previous two diagrams. The essential point to prove is again under which circumstances the reduced phase space $\left(R, \omega_{R}\right)$ is isomorphic to (or at least a covering of) an appropriate orbit space.

Theorem 4 Let $\Phi^{\alpha}, 1 \leq \alpha \leq d$ be regular constraint functions with nonempty constraint surface $C=\phi^{-1}(0)$ on a connected symplectic manifold $(M, \omega)$ with a complete Poisson $\operatorname{map} \phi:(M, \omega) \rightarrow\left(P, \Pi_{P}\right), x \mapsto \Phi^{\alpha}(x)$, and denote the symplectic leaf through $0 \in P$ by $L_{0}$ and its pre-image under $\phi$ in $M$ by $M_{0}:=\phi^{-1}\left(L_{0}\right)$. The orbit space of $M\left(M_{0}\right)$ with respect to the flow generated by the constraints $\Phi^{\alpha}$ is denoted by $Q\left(Q_{0}\right)$.

If $Q$ is a manifold, then the reduced phase space $R$ is a (symplectic) covering of $Q_{0}$, which is a symplectic leaf of $Q$. If $\pi_{1}\left(L_{0}\right)=0$, this covering is a symplectomorphism.

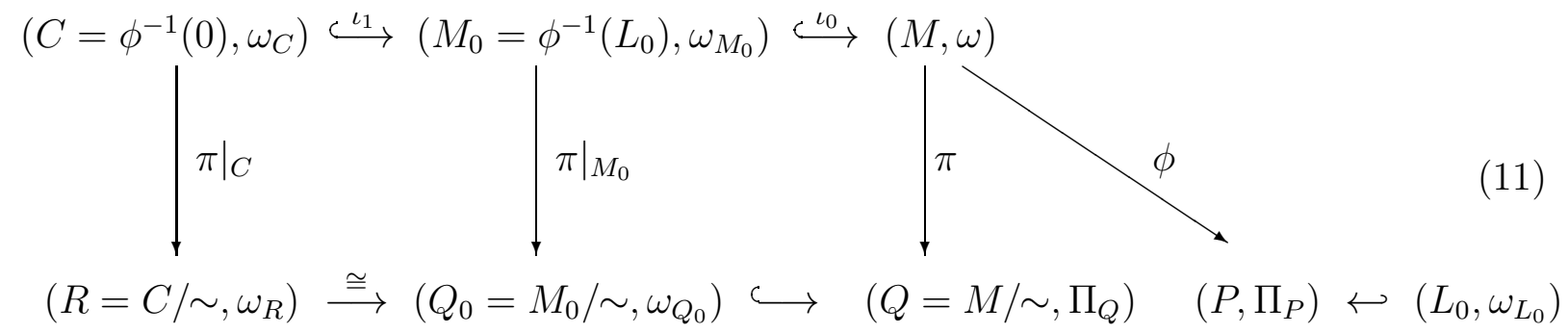

The standard reduction procedure consists in first going to the presymplectid ${ }^{\text {P }}$ constraint surface $\left(C, \omega_{C}\right)$ embedded by $\iota=\iota_{0} \circ \iota_{1}$ into $M$ and then taking the factor space with respect

\footnotetext{
${ }^{9}$ Within this subsection 'presymplectic' not necessarily contains the condition that the kernel of the closed 2-form has constant rank.
} 
to the orbits generated by the vector fields in the kernel of $\omega_{C}$. (By a slight abuse of notation we denoted the corresponding equivalence relation by $\sim$, too, while the flow of all of the constraints $\Phi^{\alpha}$ certainly does not remain inside of $C$ ). The second step is equivalent to restricting the projection map $\pi: M \rightarrow Q$ to $C \subset M$. According to the theorem (provided the covering map from $R$ to $Q_{0}$ is an isomorphism), we may alternatively restrict to a generically much larger (also presymplectic) submanifold $\left(M_{0}, \omega_{M_{0}}\right)$ of $M$ and then, in a second step, factor out the flow generated by all the constraints $\Phi^{\alpha}$. (Note that not all the vector fields $v^{\alpha}$ generating this flow are in the kernel of $\omega_{M_{0}}$. Below, however, we will define another presymplectic form $\bar{\omega}_{M_{0}}$, generalizing $\bar{\omega}$ of Corollary $\mathbb{1}$, so that the kernel of this presymplectic form is spanned by the $v^{\alpha}$.) Finally, as obvious also from the commutative diagram (11) above, a third alternative consists in factoring out the flow of all the constraints in a first step resulting in the Poisson manifold $Q$, in which $Q_{0}$ is embedded as a symplectic leaf.

In the second approach the first step amounts to solving only the first class part of the constraints. Solving the second class part of them is traded for taking the flow of all the constraints instead of just the part of the flow which remains inside $C$. Clearly for this alternative to work the closure of the constraint algebra is essential since only then the Hamiltonian vector fields of the constraints are in involution and thus generate orbits in $M$.

Proof: Locally, one can always split the constraints into first class and second class ones. This corresponds to choosing coordinates in a neighborhood of $0 \in P$ which are adapted to the leaf $L_{0}$ : First we choose arbitrary coordinates of $L_{0}$ and supplement them by additional local functions in the kernel of the Poisson tensor $\Pi$ such that, taken together, they form a coordinate system in a neighborhood of 0 . If the transition from the original coordinates $\Phi^{\alpha}$ to the adapted ones is nonsingular (i.e. with nonvanishing Jacobian), the new coordinates form local regular and irreducible constraints. By choosing the adapted coordinates we have performed a local splitting of the constraints: the coordinates of the symplectic leaf $L_{0}$ are of second class whereas the remaining coordinates are of first class.

Restricting $M$ to $M_{0}$ amounts to solving the first class part of the constraints implying that $M_{0}$ is a coisotropic submanifold of $M$. It then follows as in the purely first class case at the beginning of this subsection that $Q_{0}$ is a symplectic leaf in $Q$. Note that the codimension of $M_{0}$ in $M$ and of $L_{0}$ in $P$ always coincide due to regularity and irreducibility of the constraints, which implies that the differential of the map $\phi$ is nonvanishing.

To obtain the reduced phase space $R$, it remains to factor out the flow generated by the first class constraints and to solve the second class constraints (in a local splitting). The first part of this procedure is obviously contained in factoring out by the equivalence class $\sim$, and analogously to the purely second class case the rest of the equivalence relation serves to solve the second class constraints.

As in Theorem 3, $R$ and $Q_{0}$ are in general not identical, even if $Q_{0}$ is a manifold. In the presence of Gribov copies $Q_{0}$ is obtained from $R$ by a discrete set of identifications such that $R \rightarrow Q_{0}$ is a covering if $Q_{0}$ is a manifold (which is always the case if there is a finite number of such identifications). Using Lemma 2 one sees that $M_{0}$ is a fiber bundle 
over $L_{0}$ with a natural flat connection whose holonomy group determines the number of Gribov copies. Hence, a sufficient condition for the absence of identifications is simply connectedness of $L_{0}$.

As in Corollary 1 we may equip $M_{0}$ with a presymplectic form $\bar{\omega}_{M_{0}}$ such that its kernel spans the tangent space of the fibers of $\pi: M_{0} \rightarrow Q$. The image of $M_{0} \subset M$ under $\phi$ is $L_{0}$. Subtracting from (the presymplectic form) $\omega_{M_{0}}$ the pull back of $\omega_{L_{0}}$ under this map (i.e. under $\phi \circ \iota_{0}$ ) we obtain a presymplectic 2-form $\bar{\omega}_{M_{0}}$ such as we did in Corollary 1 . Note that the dimension of the kernel of $\bar{\omega}_{M_{0}}$ equals $d$, the number of constraints, and thus in particular is constant on all of $M_{0}$. (The proof follows along the same lines as in the symplectic case.)

As in the previous cases also here there is a Poisson perspective of the reduction process, dual to the one using either the presymplectic manifold $\left(M_{0}, \bar{\omega}_{M_{0}}\right)$ or the presymplectic manifold $\left(C, \omega_{C}\right)$.

In the particular case of $P$ being symplectic, $\iota_{0}$ becomes an isomorphism, $M_{0} \cong M$, and likewise $Q_{0} \cong Q$, so that the middle part of the diagram (11) may be dropped (disappears). Furthermore, the presymplectic form $\omega_{C}$ and the Poisson bivector $\Pi_{Q}$ become nondegenerate, rendering both spaces symplectic, $\pi_{C}$ becomes an isomorphism, and the diagram (11) reduces to the one of the previous subsection, diagram (99).

Let us finally note that the problem of Gribov copies (in the sense used in this paper) can occur only if the constrained system is not purely of first class. For first class constraints, the equivalence relation $\sim$ is exactly what is needed for the standard constraint reduction; only when there are also second class constraints can a discrete set of surplus identifications occur. The description of systems with constraints of both first and second class (or more generally of mixed type) described in the preceding paragraphs suggests to take those identifications seriously and to identify the physical phase space with $Q_{0}=M_{0} / \sim$ in all cases. The additional identifications can then be interpreted as large gauge transformations which appear together with the gauge transformations generated by first class constraints.

\subsection{Transforming second class constraints to a first class system}

In the present subsection we extend on the considerations at the end of Sec. 3.2 with the aim to reformulate the constrained system with symplectic $P$ as a first class system in an extended phase space $\widetilde{M}$. The setting is that of Corollary 2' with a simply connected $P$, the original phase space $M$ being a trivial fiber bundle with base space $R \cong C$ and fiber $P$, endowed with a canonical flat connection (Lemma Q 2 ). Moreover, with the respective two projections, $\left(C, \omega_{C}\right)$ and $\left(P, \Omega_{P}\right)$ form a symplectic dual pair with respect to $(M, \omega)$ (Theorem 3 and Corollary 2 ). The simply connectedness is a sufficient condition for the orbit space of $(M, \bar{\omega})$ to coincide with the reduced phase space $\left(C, \omega_{C}\right)$; otherwise the former can be also a covering of the latter, giving rise to 'Gribov copies'. (Cf also diagram (9). The considerations below apply also to the slightly more general case where $\pi_{1}(P) \neq 0$ but still the holonomy of the flat connection is trivial such that $Q \cong R$.) If the assumptions of Corollary 2' are fulfilled, there are no Gribov problems and the reduced phase space 
$\left(C, \omega_{C}\right)$ can be identified with the orbit space of the presymplectic manifold $(M, \bar{\omega})$ [7]. This orbit space, in turn, is obtained by symplectic reduction (using first class constraints) of an extended phase space which can be constructed in, e.g., one of the following two ways.

\subsection{1 $(M, \bar{\omega})$ as graph of the constraint map}

Let $\left(\widetilde{M}_{1}, \widetilde{\omega}_{1}\right):=(M, \omega) \times\left(P,-\Omega_{P}\right)$ and embed $M$ in $\widetilde{M}_{1}$ as the graph $\Gamma_{\phi}$ of the map $\phi: M \rightarrow P$, i.e. $M \rightarrow\{(x, \phi(x)): x \in M\} \subset \widetilde{M}_{1}$. As a direct product of two symplectic manifolds $\widetilde{M}_{1}$ is symplectic with the product symplectic structure. Note that we take $P$ with the symplectic structure reversed, i.e. $\widetilde{\omega}_{1}=\pi_{M}^{*} \omega-\pi_{P}^{*} \Omega_{P}$ where the projections from $\widetilde{M}_{1}$ to $M$ and $P$, respectively, are denoted by $\pi_{M}$ and $\pi_{P}$. This ensures that the embedding of $M$ into $\widetilde{M}_{1}$ as the graph of $\phi$ is coisotropic owing to Lemma 1, which can also be checked directly: As a subset, $M$ is specified by the constraints $\Psi^{\alpha}(x, \Phi):=\Phi^{\alpha}-\Phi^{\alpha}(x)=0$ where $\Phi^{\alpha}$ are coordinates of $P$ and $\Phi^{\alpha}(x)$ is the respective original constraint as a function on $M$. The new constraints satisfy

$$
\left\{\Psi^{\alpha}(x, \Phi), \Psi^{\beta}(x, \Phi)\right\}_{\widetilde{M}_{1}}=-\left\{\Phi^{\alpha}, \Phi^{\beta}\right\}_{P}+\left\{\Phi^{\alpha}(x), \Phi^{\beta}(x)\right\}_{M}=-\Pi_{P}^{\alpha \beta}(\Phi)+\Pi_{P}^{\alpha \beta}(\phi(x)) \approx 0
$$

by definition of $\Pi_{P}$ (" $\approx 0$ " denotes vanishing on the constraint surface). This verifies that $M$ is coisotropically embedded as the graph of $\phi$ in $\widetilde{M}_{1}$, and therefore the second class constraints $\Phi^{\alpha}$ can be replaced by first class constraints $\Psi^{\alpha}$. The constraint surface $\Psi^{\alpha}=0$ is the presymplectic manifold $(M, \bar{\omega})$ (rather than the symplectic manifold $(M, \omega)$ ) and factoring out the kernel of $\bar{\omega}$ leads to the reduced phase space $\left(R, \omega_{R}\right)$ according to Corollary 2'.

We thus obtain

Proposition 5 The reduced phase space $\left(R, \omega_{R}\right)$ of the second class constrained system $(M, \omega)$ with constraint map $\phi: M \rightarrow P, P$ symplectic and simply connected, is symplectomorphic to the reduced phase space of the first class constrained system $\left(\widetilde{M}_{1}, \widetilde{\omega}_{1}\right):=$ $(M, \omega) \times\left(P,-\Omega_{P}\right)$ with the graph of $\phi$ as constraint surface.

Note that the Poisson bracket (12) of two first class constraints $\Psi^{\alpha}$ in general vanishes only on the constraint surface $\Phi=\phi(x)$. Correspondingly, in the extended phase space $\widetilde{M}_{1}$ the new constraints are first class, but, in general, they do no longer form a closed Poisson subalgebra. In fact, they form a closed algebra, iff $\Pi_{P}$ is at most linear in $\Phi$ (while still respecting $\operatorname{det} \Pi_{P}^{\alpha \beta} \neq 0$, if necessary with a restricted $M$ ), i.e. in the case of some centrally extended Lie algebras. In contrast, the second construction introduced now, despite being more complicated at first sight, always leads to abelian (i.e. Poisson commuting) first class constraints.

\footnotetext{
${ }^{10}$ We are grateful to P. Bressler for suggesting this construction.
} 


\subsubsection{Extension by a Whitney sum}

According to Lemma 2, $M$ is a fiber bundle over $P$. So are $P$ itself (with trivial fiber) and $T^{*} P$, and we can form the Whitney sum of these bundles to obtain a new fiber bundle $\widetilde{M}_{2}:=M \oplus P \oplus T^{*} P$ with base $P$ and fiber $C \times \mathbb{R}^{d}$ :

Definition 7 Let $E_{1} \stackrel{\pi_{1}}{\longrightarrow} B$ and $E_{2} \stackrel{\pi_{2}}{\longrightarrow} B$ be two fiber bundles over the same base manifold $B$. The Whitney sum $E_{1} \oplus E_{2}$ is defined as the fiber bundle over $B$ given by

$$
E_{1} \oplus E_{2}=\left\{\left(u_{1}, u_{2}\right) \in E_{1} \times E_{2}: \pi_{1} \times \pi_{2}\left(u_{1}, u_{2}\right)=(p, p)\right\} .
$$

If the bundles $E_{1}$ and $E_{2}$ are equipped with presymplectic forms $\omega_{1}$ and $\omega_{2}$, respectively, we can also define $\left(E_{1}, \omega_{1}\right) \oplus\left(E_{2}, \omega_{2}\right)$ as a sum of presymplectic manifolds which will be equipped with a new presymplectic form:

Definition 8 Let $\left(E_{1}, \omega_{1}\right)$ and $\left(E_{2}, \omega_{2}\right)$ be presymplectic manifolds which are simultaneously fiber bundles over the same base manifold. Then the sum $\left(E_{1}, \omega_{1}\right) \oplus\left(E_{2}, \omega_{2}\right)$ is the fiber bundle $\left(E_{1} \oplus E_{2}, \omega_{1} \oplus \omega_{2}\right)$ with

$$
\omega_{1} \oplus \omega_{2}:=p_{1}^{*} \omega_{1}+p_{2}^{*} \omega_{2}
$$

where $p_{i}: E_{1} \oplus E_{2} \rightarrow E_{i}$ are the projections to the respective factors.

In our case, we have the three symplectic manifolds $(M, \omega),\left(P, \Omega_{P}\right)$ and $T^{*} P$ which all are fiber bundles over $P$. We define the extended phase space $\left(\widetilde{M}_{2}, \widetilde{\omega}_{2}\right):=(M, \omega) \oplus$ $\left(P,-\Omega_{P}\right) \oplus T^{*} P$. Note that $P$ enters with negative symplectic structure, which implies that the presymplectic form on the sum of the first two spaces is just $\bar{\omega}$ [note that $M \oplus P=M$ topologically, and the projections are $(M \oplus P \rightarrow M)=\mathrm{id},(M \oplus P \rightarrow P)=\phi]$. In addition to the projection $p_{1}: \widetilde{M}_{2} \rightarrow M$ we have a canonical embedding $i: M \rightarrow \widetilde{M}_{2}$ as the zero section in the $T^{*} P$-part of the fiber. On the extended phase space we also add the symplectic form of $T^{*} P$ which results in a symplectic form $\widetilde{\omega}_{2}$ :

Lemma 3 The form $\widetilde{\omega}_{2}=p_{1}^{*} \bar{\omega}+\mathrm{d} \Phi^{\alpha} \wedge \mathrm{d} \pi_{\alpha}$ on $\widetilde{M}_{2}=M \oplus \bar{P} \oplus T^{*} P$ is nondegenerate ( $\mathrm{d} \Phi^{\alpha} \wedge \mathrm{d} \pi_{\alpha}$ is the symplectic form on $T^{*} P$ pulled back to $\widetilde{M}_{2}$ via $p_{3}$ ).

Proof: In this proof we will denote any Hamiltonian vector field generated by a function $f$ using the symplectic structure $\omega$ on $M$ by $X_{f}$. It can be transported to vector fields on $i(M) \subset \widetilde{M}_{2}$ by push forward with the canonical embedding $i$. Choosing local coordinates $y^{i}$ on the fibers of $\phi$ and $\Phi^{\alpha}$ in $P$, we then have $\left.\left.X_{\Phi^{\alpha}}\right\lrcorner \omega=\mathrm{d} \Phi^{\alpha}, X_{y^{i}}\right\lrcorner \omega=\mathrm{d} y^{i}$ and, by definition of $\left.\Omega_{P}, X_{\Phi^{\alpha}}\right\lrcorner \mathrm{d} \Phi^{\beta}=\left\{\Phi^{\beta}, \Phi^{\alpha}\right\}=\left(\Omega_{P}^{-1}\right)^{\alpha \beta}$. As already seen in Corollary $\left.\mathbb{1}, X_{\Phi^{\alpha}}\right\lrcorner \bar{\omega}=0$ and thus

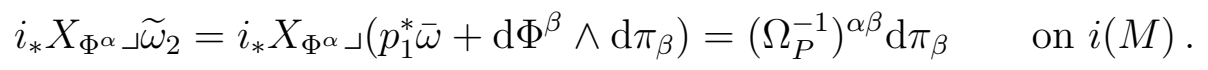

Since $\left.\partial / \partial y^{i}\right\lrcorner\left(\widetilde{\omega}_{2}-\omega\right)=0$,

$$
\left.i_{*} X_{y^{i}}\right\lrcorner \widetilde{\omega}_{2}=\mathrm{d} y^{i}+m_{i \alpha} \mathrm{d} \Phi^{\alpha}+n_{i}^{\alpha} \mathrm{d} \pi_{\alpha} \quad \text { on } i(M)
$$


with some coefficient functions $m_{i \alpha}$ and $n_{i}^{\alpha}$. Finally, on all of $\widetilde{M}$ (not just on $i(M)$ )

$$
\left.\frac{\partial}{\partial \pi_{\alpha}}\right\lrcorner \widetilde{\omega}_{2}=-\mathrm{d} \Phi^{\alpha}
$$

which together with (13) and (14) demonstrates that $\widetilde{\omega}_{2}^{\#}: T \widetilde{M} \rightarrow T^{*} \widetilde{M}$ is surjective and hence (due to equal dimension of domain of definition and image) bijective on $i(M)$. By continuity of $\widetilde{\omega}_{2}$, this holds true in a neighborhood of $i(M)$, and translation invariance of $\widetilde{\omega}_{2}$ in the $\pi_{\alpha}$-directions (according to (15), $\partial / \partial \pi_{\alpha}$ are Hamiltonian vector fields) implies that $\widetilde{\omega}_{2}$ is nondegenerate on all of $\widetilde{M}_{2}$.

Important in physical applications is also the following observation:

Lemma 4 Let $\pi_{\alpha}, 1 \leq \alpha \leq d$ denote the pull back by $p_{3}: \widetilde{M}_{2} \rightarrow T^{*} P$ of the momenta on $T^{*} P$. Then $\left\{\pi_{\alpha}, \pi_{\beta}\right\}=0$ on $\left(\widetilde{M}_{2}, \widetilde{\omega}_{2}\right)$.

Proof: According to (13), on the constraint surface $i(M)$ the variable $\pi_{\alpha}$ generates the Hamiltonian vector field $X_{\pi_{\alpha}}=\left(\Omega_{P}\right)_{\alpha \beta} i_{*} X_{\Phi^{\beta}}$ (where $X_{\Phi^{\alpha}}$ is the vector field generated by $\Phi^{\alpha}$ using $\omega$ on $\left.M\right)$ using the symplectic structure $\widetilde{\omega}_{2}$. Therefore, $\left.\left\{\pi_{\alpha}, \pi_{\beta}\right\}\right|_{i(M)}=$ $i_{*} X_{\pi_{\beta}} \pi_{\alpha}=0$. Since $\partial / \partial \pi_{\alpha}$ are Hamiltonian vector fields on $\widetilde{M}_{2}$, we have $\mathcal{L}_{\partial / \partial \pi_{\gamma}}\left\{\pi_{\alpha}, \pi_{\beta}\right\}=$ $\left\{\mathcal{L}_{\partial / \partial \pi_{\gamma}} \pi_{\alpha}, \pi_{\beta}\right\}+\left\{\pi_{\alpha}, \mathcal{L}_{\partial / \partial \pi_{\gamma}} \pi_{\beta}\right\}=0$, which implies $\left\{\pi_{\alpha}, \pi_{\beta}\right\}=0$ on all of $\widetilde{M}_{2}$.

Collecting the results we obtain

Proposition 6 The reduced phase space $\left(R, \omega_{R}\right)$ of the second class constrained system $(M, \omega)$ with constraint map $\phi: M \rightarrow P, P$ symplectic and simply connected, is symplectomorphic to the reduced phase space of the constrained system $\left(\widetilde{M}_{2}, \widetilde{\omega}_{2}\right):=(M, \omega) \oplus \bar{P} \oplus T^{*} P$ with the momenta of $T^{*} P$ as abelian first class constraints.

\subsubsection{Possible application and generalization}

On $\widetilde{M}_{2}$ we can use local coordinates $\left(x^{i}, \pi_{\alpha}\right)$ in which the symplectic form is $\widetilde{\omega}_{2}(x, \pi)=$ $\bar{\omega}(x)+\mathrm{d} \Phi^{\alpha}(x) \wedge \mathrm{d} \pi_{\alpha}$ and the constraints are just $\pi_{\alpha}=0$. (Note that by construction $\widetilde{M}_{2}$ is $M \times \mathbb{R}^{d}$ globally if and only if $T^{*} P \cong P \times \mathbb{R}^{d}$, i.e., if $P$ is parallelizable.) Despite of the simple appearance of $\pi_{\alpha}$ in the symplectic form, it is quite nontrivial that the constraints Poisson commute (on all of $\widetilde{M}_{2}$ ). In comparison, the first extension $\widetilde{M}_{1}$ is always a product manifold $\widetilde{M}_{1} \cong M \times P$, albeit with a topologically more complicated factor ( $P$ may have nontrivial topology, even if embedded into $\mathbb{R}^{d}$ ). This extension, however, has nonabelian first class constraints, in general they may even have nontrivial structure functions. Various quantization schemes, such as BRST quantization (cf., e.g., 30]), simplify greatly in the case of Poisson commuting constraints.

In both approaches the original second class system is reformulated as a first class system. The original constraint surface $C \cong R$ is one admissible gauge fixing surface in 
the new system. Choosing this gauge, i.e. $\Phi^{\alpha}(x)=0$, in a quantization scheme relying on a gauge fixation, such as some path integral quantization schemes (Faddeev-Popov procedure and its BRST- or BV-generalization), one reobtains the path integral formulation of the original second class system (as one may verify for both approaches by straightforward explicit calculations). However, now one has the option to choose another gauge, which may greatly simplify the resulting path integral measure in concrete applications. Or one can use a quantization scheme that does not need any gauge fixing, such as Dirac quantization [1] (cf. also [30]), where one regards physical states as those which are in the kernel of the quantum operators corresponding to the constraint functions; this scheme is applicable only for a first class constraint system (the first class property remaining alive even after quantization, i.e. assuming the absence of anomalies).

The reformulation in Proposition 6 may be generalized by relaxing the constraints $\pi_{\alpha} \approx 0$ to, e.g., $\pi_{\alpha}-f_{\alpha}(x) \approx 0$ for some choice of $f: M \rightarrow P$ such that the new system of constraints is still first class in $\left(\widetilde{M}_{2}, \widetilde{\omega}_{2}\right)$. It is possible that such more complicated constraints lead to a simplification in the final result for a path integral measure after fixing the gauge.

It does not seem straightforward (although interesting) to us how to extend the first class reformulation of the present subsection to the general case of a Poisson manifold $P$ (not necessarily symplectic). If, on the other hand, the constraints $\Phi^{\alpha}$ may be split globally (on all of $M$, not just in a neighborhood of $C$ ) into first class and second class constraints, then essentially in the above extension one merely has to replace $\left(P, \Omega_{P}\right)$ by the symplectic leaf $\left(L_{0}, \omega_{L_{0}}\right)$ and the extension goes through without difficulties.

In order to transform a complete physical system from second class to first class, one also has to find observables (in particular this applies to the Hamiltonian) which are in involution with the new constraints. In general, this can be done as follows: Observables of second class systems are arbitrary functions on $M$, where, however, only the restriction to the constraint surface is of physical significance. This means that independent observables correspond to arbitrary functions on $Q$. Using the projection $\pi$ they can be pulled back to $M$ and then extended in an arbitrary way to the extended phase space to yield a set of functions there. By construction, these functions are in involution with the new constraints on the 'constraint surface' $M$ (they are constant on the orbits of $M$ ) and so are observables of the first class system. See also [31 for a discussion of observables in a nonstandard approach of handling second class constrained systems.

The two proposals for turning a second class constrained system into a first class one may be compared to other methods in the literature. First, there is the Faddeev-Shatashvili approach [16] of handling second class constraints. In fact, for their toy model $(M \cong$ $\left.T^{*}\left(\mathbb{R}^{2}\right), \mathrm{d} x \wedge \mathrm{d} p_{x}+\mathrm{d} y \wedge \mathrm{d} p_{y}\right)$ constrained by $\Phi^{1}=-x, \Phi^{2}=-p_{x}$, Prop. 5 specializes to [16]. However, for their more complicated, realistic system of physical interest, the constructions differ on several grounds: There are, e.g., topological differences between the extended phase spaces; but above all, their original system of second class constraints does not form a closed Poisson subalgebra, and thus the constructions developed in the present paper (and also in [0]) do not even apply. 
Another method for turning a second class constrained system into a first class one has been introduced in [17]. Besides working only locally, the new first class constraints are given there by an iteration procedure which does not allow to find the constraints in closed form for a general system. An alternative has been mentioned in [18] where the extended phase space is simply $\widetilde{M}=M \times \mathbb{R}^{d}$ with symplectic form $\widetilde{\omega}=p_{1}^{*} \omega_{M}+\left(p_{2}^{*} \mathrm{~d} \pi_{\alpha}\right) \wedge\left(p_{1}^{*} \mathrm{~d} \Phi^{\alpha}\right)$ (in our notation, denoting the projections from $\widetilde{M}$ to $M$ and $\mathbb{R}^{d}$ by $p_{1}$ and $p_{2}$, respectively; $\pi_{\alpha}$ are global coordinates of $\mathbb{R}^{d}$ ). This procedure obviously works globally, and it also turns the second class constraints $\Phi^{\alpha}$ into abelian first class constraints $p_{1}^{*} \Phi^{\alpha}$ on $\widetilde{M}$ (the fact that the constraints on $\widetilde{M}$ are abelian follows easily from $\left.v^{\alpha}=X_{p_{1}^{*} \Phi^{\alpha}}=\frac{\partial}{\partial\left(p_{2}^{*} \pi_{\alpha}\right)}\right)$. However, here we prefer the extension $\widetilde{M}_{2}$, which also leads to an abelian first class system, because according to (15) it preserves the orbits generated by the original second class constraints on $M$, whereas the method of [18] leads to orbits $\mathbb{R}^{d}$ in the constraint surface which are always of trivial topology. (Note that in this extension it is not possible to choose $p_{2}^{*} \pi_{\alpha}$ as first class constraints with $\Phi^{\alpha}=0$ as gauge fixing conditions since the constraint manifold $p_{2}^{*} \pi_{\alpha}=0$ would be $(M, \omega)$ and so nondegenerate.)

\section{Dirac brackets and leaf-symplectic embeddings of Poisson manifolds}

In the previous section we have seen the appearance of Poisson manifolds in the context of a constrained system with closed constraint algebra. In the pure second class case, however, both $P$ and $Q$ were symplectic. On the other hand, we noted that the original manifold $M$ can be equipped naturally with a presymplectic form (\$) such that, under favorable circumstances specified above which guaranteed the absence of a 'Gribov problem', the constraint surface is an admissible cross section of the respective orbits.

There is, however, another perspective, in a way dual to the one with a presymplectic manifold $(M, \bar{\omega})$ and which is applicable for any second class constrained system (not necessarily forming a closed algebra), where a Poisson manifold plays a role. This is the concept of the well-known Dirac bracket [1].

\subsection{Dirac bracket}

Given a symplectic manifold $(M, \omega)$ with a system of (regular, irreducible) second class constraints $\Phi^{\alpha}, \alpha=1, \ldots, d,\left\{\Phi^{\alpha}, \Phi^{\beta}\right\}(x)=F^{\alpha \beta}(x)$, $\operatorname{det} F^{\alpha \beta} \not \approx 0$, Dirac defined the so called Dirac bracket as a modified Poisson bracket $\{\cdot, \cdot\}_{D}$ on $M$. We again denote the constraint surface $\Phi^{\alpha}(x)=0$ by $C$ and the induced symplectic 2 -form by $\omega_{C}$; if $\iota_{C}: C \hookrightarrow M$ is the respective embedding map, then $\omega_{C}=\iota_{C}^{*} \omega$. Using the Poisson bivector $\Pi$ inverse to $\omega$, the bivector $\Pi_{D}$ corresponding to the bracket $\{\cdot, \cdot\}_{D}$ has the form

$$
\Pi_{D}=\Pi+\frac{1}{2} G_{\alpha \beta} v^{\alpha} \wedge v^{\beta}
$$


where $G_{\alpha \beta}$ is the inverse to $F^{\alpha \beta}$, well-defined at least in some tubular neighborhood $S \subset M$ of the constraint surface, $C \subset S$, and $v^{\alpha}=\left\{\cdot, \Phi^{\alpha}\right\} \equiv-\left(\partial_{i} \Phi^{\alpha}\right) \Pi^{i j} \partial_{j}$ is the Hamiltonian vector field generated by the constraint $\Phi^{\alpha}$.

One may verify that $\Pi_{D}$ indeed satisfies the Jacobi identity and thus defines a Poisson bracket.⿴囗十 By construction the constraint functions restricted to $S,\left.\Phi^{\alpha}\right|_{S} \in C^{\infty}(S)$, span the center of the algebra generated by this bracket. Correspondingly the constraint surface is a symplectic leaf of $\Pi_{D}$. Moreover, the symplectic form on that leaf coincides with $\omega_{C}$ (we will verify this explicitly in Proposition 0 below.)

Inspired by the relation of the Dirac bracket of a second class constrained system with the original symplectic structure, we make the following definition.

Definition 9 Let $(P, \Pi)$ be a Poisson manifold and $\Omega_{L}$ denote the induced symplectic form on a given leaf $L$ of the stratification of $P$ with $\iota_{L}: L \hookrightarrow P$ being the respective embedding map. A (symplectic, presymplectic,...) 2-form $\widetilde{\Omega}$ and a Poisson bivector $\Pi$ are called compatible within $S \subset P$, if for any leaf $L$ in $S: \Omega_{L}=\iota_{L}^{*} \widetilde{\Omega}$.

Remark: There is an obvious adaptation of the above definition to the case of an almost Poisson bivector $\Pi$ which still generates a foliation (stratification) - so called $H$-Poisson or twisted Poisson structures $\Pi$, fulfilling $[\Pi, \Pi]=\left(\Pi^{\sharp}\right)^{\otimes 3} H$ for some closed 3-form $H$ 32, 33, 34, 35.

The Dirac bivector (16) is well-defined in some subset $S \subset M$ containing the constraint surface $C$. Within $S$ we have the following relation

Proposition 7 The Dirac bivector $\Pi_{D}$ and the symplectic form $\omega \in \Lambda^{2}\left(T^{*} M\right)$ are compatible within the domain $S \subset M$ of definition of $\Pi_{D}$.

Proof: It suffices to show that for any two functions $f$ and $g$ on a given leaf $L$ of the foliation with embedding map $\iota_{L}: L \rightarrow S$ one has on all of $L$

$$
\iota_{L}^{*}\left(\{F, G\}_{D}\right)=\tilde{X}_{g}(f),
$$

where $F$ and $G$ are arbitrary extensions of $f$ and $g$ to $S$, such that $\iota_{L}^{*} F=f$ and $\iota_{L}^{*} G=g$, and where $\widetilde{X}_{g} \in \Gamma(T L)$ is (uniquely) defined by means of $\left.\widetilde{X}_{g}\right\lrcorner \iota_{L}^{*} \omega=\mathrm{d} g$. (The right hand side is the Poisson bracket between $f$ and $g$ as induced by $\iota_{L}^{*} \omega$, while on the other hand the left-hand side gives the corresponding bracket induced by the symplectic form of the

\footnotetext{
${ }^{11}$ This may be infered also from the considerations to follow. With Prop. 0 one finds that the leaves $\Phi^{\alpha}=$ const of the local foliation generated by $\Pi_{D}$ are symplectic which shows that $\Pi_{D}$ is Poisson: In a neighborhood $S$ of $C, \Pi_{D}$ has constant rank and is surface forming. This implies [35] that there is a 3-form $H$ on $S$ such that $\left[\Pi_{D}, \Pi_{D}\right]=\left(\Pi_{D}^{\sharp}\right)^{\otimes 3} H$ (such a bivector $\Pi_{D}$ is called $H$-Poisson, cf. also the remark after Def. 9). On the leaves of $\Pi_{D}$, the exterior derivative of its inverse equals the pull back of $H$. Using our later result (Prop. 7) this implies that the pull back of $H$ to an arbitrary leaf in $S$ vanishes, and so $H$ is purely transversal with respect to the foliation. The transversal part, however, is projected out by $\left(\Pi_{D}^{\sharp}\right)^{\otimes 3}$ which gives us $\left[\Pi_{D}, \Pi_{D}\right]=0$.
} 
bivector $\Pi_{D}$ on $L$. Thus, equality for all functions $f$ and $g$ proves equality of the respective 2 -forms.)

We first note that the choice of the extension of the function $f$ (and likewise of $g$ ) does not enter the left-hand side of Eq. (17), since by construction $\left\{\cdot, \Phi^{\alpha}\right\}_{D} \equiv 0$, while two different extensions $F, F^{\prime}$ of $f$ differ only by a combination of the constraints: $F^{\prime}=$ $F+c^{\alpha} \Phi_{\alpha}$ for some smooth functions $c^{\alpha}$ defined in a neighborhood of $L$ (cf. e.g. [30]). We thus may choose a particularly convenient extension, namely one such that $\iota_{L}^{*}\left(v^{\alpha}(F)\right)=0$ (and likewise for $G$ ), where, as before $v^{\alpha}=\left\{\cdot, \Phi^{\alpha}\right\}$. It then immediately follows from the definition (16) of the Dirac bracket that $\iota_{L}^{*}\{F, G\}_{D}=\left.\iota_{L}^{*}\{F, G\} \equiv \iota_{L}^{*}\left(X_{G}(F)\right) \equiv X_{G}\right|_{L}(f)$ where $X_{G}=\{\cdot, G\}$, since, due to the chosen extension, $X_{G}$ is tangential to $L$ and thus may be restricted consistently to $L$. From $\left.\left.\left.X_{G}\right|_{L}\right\lrcorner \iota_{L}^{*} \omega=\iota_{L}^{*}\left(X_{G}\right\lrcorner \omega\right)=\iota_{L}^{*} \mathrm{~d} G \equiv \mathrm{d} g$ it thus follows that $\left.X_{G}\right|_{L}=\widetilde{X}_{g}$, which proves the assertion.

\section{Remarks:}

i) The constraint surface is the preimage of the origin of the constraint map $\Phi^{\alpha}: M \rightarrow$ $\mathbb{R}^{d}$. (Note that we do here no longer require that the constraint algebra is closed; thus, the target does not inherit canonically a Poisson bracket in this case). This corresponds only to one particular symplectic leaf $L_{0}$ of $\left(S, \Pi_{D}\right)$. If one shifts the constraint surface slightly by setting $\Phi^{\alpha}(x)$ to some constant $c^{\alpha} \in \mathbb{R}^{d}$ small enough such that the respective preimage $L_{c}$ is still in $S$, the bivector $\Pi_{D}$ given in (16) still provides the respective Dirac bracket. So all the leaves $L_{c}$ of $\left(S, \Pi_{D}\right)$ are seen to be possible constraint surfaces.

ii) Constraints describing a fixed constraint surface $L_{0}$ are defined only up to redefinitions $\Phi^{\alpha} \rightarrow \widetilde{\Phi}^{\alpha} \equiv A_{\beta}^{\alpha} \Phi^{\beta}$ of the constraint functions, where the coefficient matrix $A_{\beta}^{\alpha}(x)$, required to have a nonvanishing determinant on $C$, is in general a smooth function on phase space (or at least on some neighborhood of $C$ ). The corresponding Dirac bivector $\widetilde{\Pi}_{D}$, defined in some region $\widetilde{S} \subset M$ containing $C$, now has in general different symplectic leaves on the intersection of $S$ and $\widetilde{S}$ to the ones generated by $\left(\Pi_{D}, S\right)$; in general only the constraint surface $C$ itself is a joint symplectic leaf. So, one obtains different possible constraint surfaces $\widetilde{L}_{c}$, shifted as in the previous remark, when one redefines the constraint functions which specify the original constraint surface $C=L_{0}=\widetilde{L}_{0}$. Any Dirac bivector $\Pi_{D}$ in Eq. (16), corresponding to some specific choice of the constraint functions $\Phi^{\alpha}$ and defined in a region $S \subset M$, is compatible with the original symplectic 2-form $\omega$ within $S$.

iii) According to the above consideration, $S$ is foliated into leaves $C$ (with a slight abuse of notation, since previously $C$ denoted only the original constraint surface) which are all second class submanifolds, and at any point $x \in S \subset M$ we have a splitting of the tangent space according to $T_{x} M=T_{x} C \oplus T_{x} C^{\perp}$, and likewise of the cotangent bundle $T_{x}^{*} M=\operatorname{Ann}_{M}\left(T_{x} C^{\perp}\right) \oplus \operatorname{Ann}_{M}\left(T_{x} C\right)$, induced by the original bivector $\Pi$ (cf. also (1) with $\omega^{\sharp}=-\left(\Pi^{\sharp}\right)^{-1}$ and recall the discussion at the end of the proof of Theorem 3). Denote by $\pi_{1}$ the projection to the first factor in $T_{x} M$ and by $\bar{\pi}_{1}$ to the first factor in $T_{x}^{*} M$ and regard both $\Pi$ and $\Pi_{D}$ as bivector fields defined on $S \subset M$. Then the definition (16) of the Dirac bivector $\Pi_{D}$ is equivalent [36] to saying that $\Pi_{D}$ is the projection of $\Pi$ to $T C$ along $T C^{\perp}$. In formulas this becomes $\Pi_{D}^{\sharp}=\pi_{1} \circ \Pi^{\sharp} \circ \bar{\pi}_{1}$ or, equivalently, $\Pi_{D}=\Pi \circ\left(\bar{\pi}_{1} \otimes \bar{\pi}_{1}\right)$ or, if one regards 
the bivectors as elements or sections of $\Lambda^{2} T M, \Pi_{D}=\left(\pi_{1} \otimes \pi_{1}\right) \Pi$. This follows easily using previous results: As mentioned before Prop. 1, we have $\operatorname{Ann}_{M}(T C)=\prec \mathrm{d} \Phi^{\alpha} \succ$ implying $\pi_{1} \circ \Pi^{\sharp} \circ \bar{\pi}_{1}\left(\mathrm{~d} \Phi^{\alpha}\right)=0$. Furthermore, $\pi_{1} \circ \Pi^{\sharp} \circ \bar{\pi}_{1}$ restricted to the leaves is by definition of $\bar{\pi}_{1}$ non-degenerate and coincides with $\Pi^{\sharp}$. So the two properties which characterize the Dirac bivector $\Pi_{D}$ in (16) are fulfilled which proves $\pi_{1} \circ \Pi^{\sharp} \circ \bar{\pi}_{1}=\Pi_{D}^{\sharp}$. This presents an alternative proof for the compatibility of $\Pi_{D}$ with the original symlectic form $\omega$.

\subsection{Closed constraint algebra revisited}

Before proceeding let us specialize the above considerations to a system of a closed, second class constraint algebra. In this case the Dirac bivector takes the form

$$
\left.\left.\Pi_{D}=\Pi-\frac{1}{2}\left(\Omega_{P}\right)_{\alpha \beta}\left(\mathrm{d} \Phi^{\alpha}\right\lrcorner \Pi\right) \wedge\left(\mathrm{d} \Phi^{\beta}\right\lrcorner \Pi\right),
$$

wherever $\Omega_{P}^{\sharp}=-\left(\Pi_{P}^{\sharp}\right)^{-1}$ is defined, i.e. at least in a tubular neighborhood $S$ of the constraint surface $C$. For simplicity we assume $S=M$, or, equivalently, $\left(P, \Pi_{P}\right)$ symplectic. (We refer to diagram (9) for the notation.) Then $\left(M, \Pi_{D}\right)$ is a Poisson manifold.

First of all we note that the expression (18) obviously is invariant with respect to a (regular) coordinate change in $P, \Phi^{\alpha} \rightarrow \widetilde{\Phi}^{\alpha}(\Phi)$, corresponding to a particular change of constraint functions, i.e. to a particular matrix $A_{\beta}^{\alpha}(x)$ in Remark ii) above. By construction, the reduced phase space $\left(R=\phi^{-1}(0), \omega_{R}\right)$ is a symplectic leaf of this Poisson manifold, furthermore. This also applies to any other, shifted value for the constraint functions, $\Phi^{\alpha}:=$ const $^{\alpha}$, cf. Remark i) above.

The bivector $\Pi_{D}$, defined on all of $M$, is not only compatible with $\omega$ (cf. Proposition $\square$ above), but clearly also with $\bar{\omega}$ defined in (8). As remarked already at the beginning of this section, $M$ equipped with the presymplectic form $\bar{\omega}$ provides a perspective 'dual' to the one following from $M$ being equipped with the Dirac bivector $\Pi_{D}$.

There is now also another (nonsymplectic) Poisson manifold we can associate to the system at hand: In Sec. 3.4, and under the assumptions stated there, we considered two reformulations of the original second class constrained system in an extended phase space. In the second of those extensions, $\left(\widetilde{M}_{2}, \widetilde{\omega}_{2}\right)$, the first class constraint algebra in the extended phase space was abelian and thus in particular closed. Therefore we can adapt diagram (10) to the present situation (combining it with part of diagram (9)):

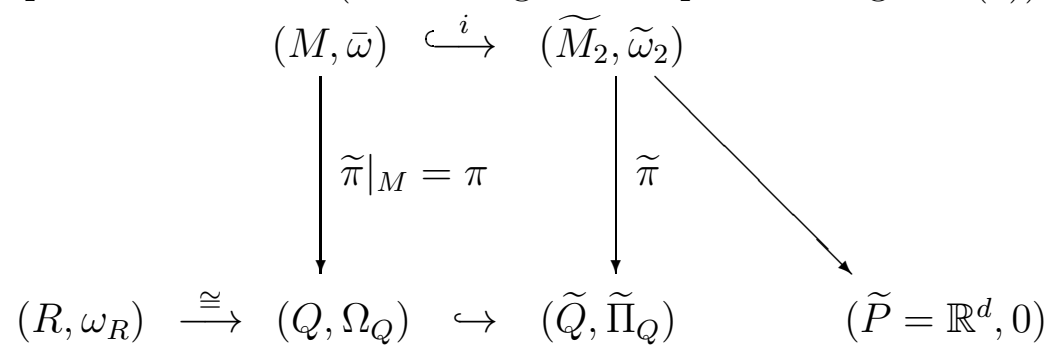

This plot displays a 'duality' between $(M, \bar{\omega})$, a presymplectic manifold, and the Poisson manifold $\left(\widetilde{Q}, \widetilde{\Pi}_{Q}\right)$. It is therefore natural to ask, what is the relation between the two 
Poisson manifolds $\left(M, \Pi_{D}\right)$ and $\left(\widetilde{Q}, \widetilde{\Pi}_{Q}\right)$. Note as an aside that the second Poisson manifold can be defined in general only by the second of the two extensions of Sec. 3.4. (As remarked at the end of Sec. 3.4.1, the first class constraints in $\widetilde{M}_{1}$ form a closed algebra only when the original constraint algebra $\Pi_{P}$ is at most linear in the constraints $\Phi^{\alpha}$.) In this physically particularly interesting case, however, an analogous question may be posed.

Proposition 8 Under the conditions specified in Corollary 2', the Poisson manifolds $\left(M, \Pi_{D}\right)$ and $\left(\widetilde{Q}, \widetilde{\Pi}_{Q}\right)$ are isomorphic, iff $P \cong \mathbb{R}^{d}$ topologically.

Proof: The necessity follows from the remark that $\widetilde{Q} \cong Q \times \mathbb{R}^{d}$, i.e. the constraints $\pi_{\alpha}$ act trivially on the local linear factor $\mathbb{R}^{d}$ in $\widetilde{M}_{2}$ (with its coordinates $\pi_{\alpha}$ ) and have a flow agreeing with that generated by $\left.\mathrm{d} \Phi^{\alpha}\right\lrcorner \Pi$ on $M$. This is a consequence of (13), where $i(M)$

may as well be taken to denote any section in $\widetilde{M}_{2}$ specified by some constant value of the momenta $\pi_{\alpha}$ (cf. also Lemma 4).

The rest of the claim is proved by establishing symplectomorphisms between the symplectic leaves $\pi_{\alpha}=$ const of $\widetilde{\Pi}_{Q}$ and $\Phi^{\alpha}(x)=$ const of $\Pi_{D}$. Using the direct product structure of $M$ under the conditions of Corollary 2', one easily sees that the leaves are all symplectomorphic to the reduced phase space, while the leaf spaces are both $\mathbb{R}^{d}$.

\subsection{Compatible presymplectic forms on neighborhoods of regu- lar leaves}

We may now reverse the question addressed originally by Dirac: Given a Poisson bivector $\Pi \in \Lambda^{2}(T M)$ on a manifold $M$, is there a symplectic (or, since $\operatorname{dim} M$ may be odd, at least presymplectic) 2 -form which is compatible with $\Pi$ in $M$ ?

In general, such a compatible presymplectic 2 -form will not exist on all of $M$ (recall that also the Dirac bivector is defined only in a neighborhood of the constraint surface); but it may nevertheless exist in some neighborhood $S$ of a given leaf $L$ in $M$. In particular rank $\Pi$ should be constant in all of $S$, so $S$ should be a regular Poisson manifold. For simplicity, we will assume that already $M$ is regular and look for a compatible presymplectic form on all of $M$.

We remark that regularity of $(M, \Pi)$ does not imply that $M$ is foliated regularly: Consider e.g. the Poisson tensor $\Pi=\left(\partial_{1}+\omega \partial_{2}\right) \wedge\left(\partial_{3}+\bar{\omega} \partial_{4}\right)$ with irrational $\omega$, $\bar{\omega}$ on a torus $T^{4}$ (coordinates $\left.x^{i} \equiv x^{i}+1\right)$. Any of its symplectic leaves is dense in all of $T^{4}$. This bivector is easily verified to permit the compatible symplectic 2 -form $\Omega=$ $(1+\omega \bar{\omega})^{-1}\left(\mathrm{~d} x_{1} \wedge \mathrm{d} x_{3}+\mathrm{d} x_{2} \wedge \mathrm{d} x_{4}\right)$ on $T^{4}$ (or simply $\mathrm{d} x_{1} \wedge \mathrm{d} x_{3}$ as a compatible presymplectic form).

We first construct an almost presymplectic form (i.e. just any 2-form, not necessarily closed) which is compatible with $\Pi$. This is possible always: Picking an auxiliary Riemannian metric on $M$, we can define a 2 -form $\widetilde{\Omega} \in \Lambda^{2}\left(T^{*} M\right)$ by requiring that for any $X \in M$ a vector normal to the leaf through $X$ is annihilated by $\widetilde{\Omega}$ whereas for a tangential vector 
$v \in T_{X} L$ we have $\left.\left.v\right\lrcorner \widetilde{\Omega}=v\right\lrcorner \Omega_{L}$ (again, $\Omega_{L}$ is the symplectic form induced on the leaf by $\Pi)$. In this way we obtain a well-defined 2 -form on $M$ which is compatible with $\Pi$, but not closed in general.

There is still some freedom in defining this form since one can add an arbitrary 2 -form $\lambda$ which vanishes when pulled back to a leaf. The space of such $k$-forms is denoted by $\Lambda_{0}^{k}(M)$. This may be exploited to obtain another compatible 2 -form $\Omega:=\widetilde{\Omega}+\lambda$. Since $\mathrm{d}$ maps $\Lambda_{0}^{k}(M)$ to $\Lambda_{0}^{k+1}(M)$ and $\mathrm{d} \widetilde{\Omega} \in \Lambda_{0}^{3}(M)$, the question of whether $\lambda$ may be chosen in such a way that $\Omega$ is closed, relates to the so-called characteristic form-class of $\Pi$, i.e. by $\mathrm{d} \widetilde{\Omega}$ regarded as an element of the relative cohomology $H_{\text {rel }}^{3}:=\operatorname{ker~}_{0}^{(3)} / \operatorname{Im~d}_{0}^{(3)}\left(\mathrm{d}_{0}^{(k)}\right.$ denotes the restriction of d to $\Lambda_{0}^{k}(M)$; cf. also [9]):

Proposition 9 Let $(M, \Pi)$ be a regular Poisson manifold and $\widetilde{\Omega}$ be any 2-form compatible with $\Pi$ (such a 2-form exists always).

There is a compatible presymplectic form $\Omega$ on $M$ if and only if the characteristic form-class of $\Pi,[\mathrm{d} \widetilde{\Omega}] \in H_{\mathrm{rel}}^{3}$, vanishes.

Proof: Note first that by construction $\mathrm{d} \widetilde{\Omega} \in \Lambda_{0}^{3}$ such that $[\mathrm{d} \widetilde{\Omega}]$ is well defined in the relative cohomology.

Let us first prove that the condition is necessary: assume that there exists a compatible presymplectic form $\Omega$. Then $[\mathrm{d} \Omega]=0$ because $\Omega$ is closed, and $\Omega=\widetilde{\Omega}+\lambda$ for some $\lambda \in \Lambda_{0}^{2}(M)$ implies $[\mathrm{d} \widetilde{\Omega}]=[\mathrm{d} \Omega]-[\mathrm{d} \lambda]=0$.

Conversely, if $[\mathrm{d} \widetilde{\Omega}]=0$, then $\mathrm{d} \widetilde{\Omega}=\mathrm{d} \lambda$ for some $\lambda \in \Lambda_{0}^{2}(M)$. This implies that $\Omega:=\widetilde{\Omega}-\lambda$ is closed and compatible.

In the remainder of this section we will discuss this main condition in a more explicit form. Using the auxiliary metric, we have a splitting of the tangent bundle $T M=T L \oplus T^{\perp} L$ interpreting both bundles on the right hand side as vector bundles over the base manifold $M$. Here, we have to assume that the normal distribution $T^{\perp} L$ is integrable for reasons which will become clear in the course of this subsection.

Using the metric, we obtain a dual decomposition of the cotangent bundle $T^{*} M$ leading to a two-fold grading of $\Lambda^{*}\left(T^{*} M\right)$ by taking exterior powers. In this way the decomposition $T M=T L \oplus T^{\perp} L$ entails a decomposition of $n$-forms $\omega \in \Lambda^{n}\left(T^{*} M\right)$. More explicitly, we have $\omega=\sum_{i=0}^{n} \omega^{(i, n-i)}$ fulfilling $\left.\left.\left.v_{j}\right\lrcorner \ldots\right\lrcorner v_{1}\right\lrcorner \omega_{X}^{(i, n-i)}=0$ for $j>n-i$ and all $v_{1}, \ldots, v_{j} \in$ $T_{X}^{\perp} L$, whereas for $j=n-i$ either $\omega^{(i, n-i)}=0$ or there are $v_{1}, \ldots, v_{j} \in T_{X}^{\perp}$ such that $\left.\left.\left.v_{j}\right\lrcorner \cdots\right\lrcorner v_{1}\right\lrcorner \omega_{X}^{(i, n-i)} \neq 0$. The uniqueness of this decomposition (for a fixed Riemannian metric) can also be seen in local coordinates adapted to the foliation of $M$, in which it amounts to collecting terms with a fixed number of differentials along normal coordinates.

Definition 10 An $n$-form $\omega$ is called pure of degree $(i, n-i)$ if it has the decomposition $\omega=\omega^{(i, n-i)}$. The space $\Lambda^{(i, n-i)}\left(T^{*} M\right) \subset \Lambda^{n}\left(T^{*} M\right)$ is the space of pure forms of degree $(i, n-i)$. 
Note that these and the following definitions depend on the Riemannian metric chosen on $M$.

We can similarly decompose the exterior derivative operator d into two parts

$$
\mathrm{d}_{\|}: \Lambda^{(i, n-i)}\left(T^{*} M\right) \rightarrow \Lambda^{(i+1, n-i)}\left(T^{*} M\right) \text { and } \mathrm{d}_{\perp}: \Lambda^{(i, n-i)}\left(T^{*} M\right) \rightarrow \Lambda^{(i, n+1-i)}\left(T^{*} M\right) .
$$

Given a pure form $\omega \in \Lambda^{(i, n-i)}\left(T^{*} M\right)$, we define

$$
\mathrm{d} \omega=(\mathrm{d} \omega)^{(i+1, n-i)}+(\mathrm{d} \omega)^{(i, n+1-i)}=: \mathrm{d}_{\|} \omega+\mathrm{d}_{\perp} \omega .
$$

Both derivative operators can be extended linearly so as to be defined on arbitrary forms. Choosing local coordinates $\left(X^{\alpha}, X^{I}\right)$ such that $X^{\alpha}$ parameterize the leaves in a neighborhood and $X^{I}$ the normal directions, the new derivative operators can be written as $\mathrm{d}_{\|}=\partial_{\alpha} \mathrm{d} X^{\alpha} \wedge$ and $\mathrm{d}_{\perp}=\partial_{I} \mathrm{~d} X^{I} \wedge$. At this point the integrability of the normal distribution has been used: otherwise there would be an additional term in the decomposition of $\mathrm{d}$ (see also [9]). This can be seen using the Cartan formula which for a 1-form $\omega$ reads

$$
\mathrm{d} \omega\left(v_{1}, v_{2}\right)=v_{1} \omega\left(v_{2}\right)-v_{2} \omega\left(v_{1}\right)-\omega\left(\left[v_{1}, v_{2}\right]\right) .
$$

Choosing $v_{1}, v_{2} \in T^{\perp} L$ such that $\left[v_{1}, v_{2}\right] \notin T^{\perp} L$ (which by definition is possible only if the normal distribution is not integrable), there is always a 1-form $\omega \in \Lambda^{(1,0)}\left(T^{*} M\right)$ with $\mathrm{d} \omega\left(v_{1}, v_{2}\right) \neq 0$. Thus, $\mathrm{d} \omega$ has a nonvanishing contribution in $\Lambda^{(0,2)}\left(T^{*} M\right)$ and $\mathrm{d} \neq \mathrm{d}_{\|}+\mathrm{d}_{\perp}$ for a nonintegrable normal distribution. One can see that there is only one additional term in the general case mapping $\Lambda^{(i, j)}\left(T^{*} M\right)$ to $\Lambda^{(i-1, j+2)}$; but this would already complicate the descent equations derived below. Therefore, we will only deal with the integrable case from now on, for which we have

\section{Lemma 5}

$$
\mathrm{d}_{\|}^{2}=0 \quad, \quad\left\{\mathrm{~d}_{\|}, \mathrm{d}_{\perp}\right\}:=\mathrm{d}_{\|} \mathrm{d}_{\perp}+\mathrm{d}_{\perp} \mathrm{d}_{\|}=0 \quad, \quad \mathrm{~d}_{\perp}^{2}=0
$$

Proof: It suffices to prove the assertion for actions on a pure form $\omega$ of arbitrary degree. We then have

$$
0=\mathrm{d}^{2} \omega=\mathrm{d}\left(\mathrm{d}_{\|} \omega+\mathrm{d}_{\perp} \omega\right)=\mathrm{d}_{\|}^{2} \omega+\left\{\mathrm{d}_{\|}, \mathrm{d}_{\perp}\right\} \omega+\mathrm{d}_{\perp}{ }^{2} \omega .
$$

Because the three terms in the sum are all pure of different degrees, they have to vanish separately.

Now we are in the position to proceed with the derivation of conditions for the existence of a compatible presymplectic form. The 2 -form $\widetilde{\Omega}$ introduced above is pure of degree $(2,0)$ by construction, $\widetilde{\Omega}=\widetilde{\Omega}^{(2,0)}$. As already discussed in the paragraph preceding Proposition 9 , $\widetilde{\Omega}$ is not necessarily closed since $\mathrm{d}_{\perp} \widetilde{\Omega} \neq 0$ in general. Adding a form $\lambda \in \Lambda_{0}^{2}(M)$ leads to a new form $\Omega=\widetilde{\Omega}^{(2,0)}+\Omega^{(1,1)}+\Omega^{(0,2)}$ which is closed if and only if

$$
\mathrm{d} \Omega=\mathrm{d}_{\perp} \widetilde{\Omega}^{(2,0)}+\mathrm{d}_{\|} \Omega^{(1,1)}+\mathrm{d}_{\perp} \Omega^{(1,1)}+\mathrm{d}_{\|} \Omega^{(0,2)}+\mathrm{d}_{\perp} \Omega^{(0,2)}=0 .
$$

Collecting forms of equal degree immediately leads to 
Proposition 10 Let $(M, \Pi)$ be a regular Poisson manifold being equipped with a Riemannian metric and an associated integrable decomposition of the tangential bundle.

There is a presymplectic 2-form $\Omega=\Omega^{(2,0)}+\Omega^{(1,1)}+\Omega^{(0,2)}$ compatible with $\Pi$ on $M$ if and only if the descent equations

$$
\mathrm{d}_{\|} \Omega^{(1,1)}=-\mathrm{d}_{\perp} \Omega^{(2,0)} \quad, \quad \mathrm{d}_{\|} \Omega^{(0,2)}=-\mathrm{d}_{\perp} \Omega^{(1,1)} \quad, \quad \mathrm{d}_{\perp} \Omega^{(0,2)}=0
$$

have a solution on $M$ subject to the condition that $\Omega^{(2,0)}$ restricted to any leaf in $M$ coincides with the symplectic form of that leaf.

In special cases of a foliation we can reformulate the conditions of the proposition.

Corollary 3 If $M$ is foliated trivially, i.e. it is of the form $M \cong L \times \mathbb{R}^{k}$, then the first equation in (20) implies

$$
\partial_{I} \oint_{\sigma} \Omega^{(2,0)}=0
$$

where $\partial_{I}$ denotes any differentiation transversal to $L$ and $\sigma$ is a closed two-cycle in $L$. This means that the symplectic volume of any closed two-cycle in a leaf has to be constant in $M$.

This condition is violated for, e.g., any family of homeomorphic coadjoint orbits of a compact, semisimple Lie algebra because the symplectic form of leaves in the dual Lie algebra with the Kirillov-Kostant structure depends nontrivially on the Casimir functions (the radial coordinate, e.g., for $s u(2)$ ). In particular, according to the work of Kirillov, the (always discrete) set of irreducible unitary representations of a compact, semisimple Lie algebra corresponds to the set of all integral symplectic leaves in the corresponding Lie Poisson manifold, that is to leaves satisfying that $\oint_{\sigma} \Omega^{(2,0)}$ is an integer multiple of a fixed constant for any two-cycle in the leaf. Correspondingly, $\oint_{\sigma} \Omega^{(2,0)}$ depends nontrivially on the leaf and Poisson manifolds of this kind do not allow compatible presymplectic forms. (In this argument we also used analyticity which implies that $\partial_{I} \oint_{\sigma} \Omega^{(2,0)}$ cannot vanish on an interval if $\oint_{\sigma} \Omega^{(2,0)}$ is not constant. This means that any open subset of the dual Lie algebra contains (part of) a leaf violating the condition of the Corollary.)

We have another interesting situation if all leaves in $M$ have trivial second cohomology: $H^{2}(L)=0$. If $M$ is foliated trivially, i.e. of the form $M \cong L \times \mathbb{R}^{k}$, one can easily verify that there is always a compatible presymplectic form:

Lemma 6 If $\widetilde{\Omega}^{(2,0)}$ has a symplectic potential $\theta^{(1,0)}$ on any leaf $L$ in $M \cong L \times \mathbb{R}^{k}$, i.e. $\widetilde{\Omega}^{(2,0)}=\mathrm{d}_{\|} \theta^{(1,0)}$, and $\theta^{(1,0)}$ varies smoothly from leaf to leaf, then $\Omega:=\mathrm{d} \theta^{(1,0)}$ is a compatible presymplectic form on $M$.

In particular, if all leaves $L$ in a trivially foliated $M \cong L \times \mathbb{R}^{k}$ have trivial second cohomology, then there exists a compatible presymplectic form on $M$. 
This can also be derived using the descent equations which acquire the form (using Lemma 5)

$$
\mathrm{d}_{\|} \Omega^{(1,1)}=\mathrm{d}_{\|} \mathrm{d}_{\perp} \theta^{(1,0)}
$$

solved by $\Omega^{(1,1)}:=\mathrm{d}_{\perp} \theta^{(1,0)}$, leading to

$$
\mathrm{d}_{\|} \Omega^{(0,2)}=-\mathrm{d}_{\perp}^{2} \theta^{(1,0)}=0
$$

in addition to $\mathrm{d}_{\perp} \Omega^{(0,2)}=0$. The latter two equations have an obvious solution $\Omega^{(0,2)}=0$ implying

$$
\Omega=\widetilde{\Omega}^{(2,0)}+\Omega^{(1,1)}=\mathrm{d}_{\|} \theta^{(1,0)}+\mathrm{d}_{\perp} \theta^{(1,0)}=\mathrm{d} \theta^{(1,0)} .
$$

As a more explicit example $\mathrm{e}^{\mathbb{T 2}}$ we look at the manifold $M=T^{2} \times \mathbb{R}$ with Poisson bivector $\Pi=F\left(x_{1}, x_{2}, x_{3}\right)\left(\partial_{1}+\omega \partial_{2}\right) \wedge \partial_{3}$ with an arbitrary function $F$ on $M$. Leaves are submanifolds subject to the condition $\omega x_{1}-x_{2}=0$ and we can use $x_{1}$ and $x_{3}$ as local coordinates of a leaf. If we choose the normal distribution to be spanned by $\partial_{2}$, any 2-form $\Omega$ is split into $\Omega=\widetilde{\Omega}^{(2,0)}+\Omega^{(1,1)}+\Omega^{(0,2)}$ with $\widetilde{\Omega}^{(2,0)}=F\left(x_{1}, x_{2}, x_{3}\right)^{-1} \mathrm{~d} x_{1} \wedge \mathrm{d} x_{3}$, $\Omega^{(1,1)}=\mu_{1} \mathrm{~d} x_{1} \wedge \mathrm{d} x_{2}+\mu_{2} \mathrm{~d} x_{3} \wedge \mathrm{d} x_{2}, \Omega^{(0,2)}=0$, and only the first descent equation is nontrivial and takes the form

$$
\mathrm{d}_{\|} \Omega^{(1,1)}=\left(\partial_{3} \mu_{1}-\partial_{1} \mu_{2}\right) \mathrm{d} x_{1} \wedge \mathrm{d} x_{2} \wedge \mathrm{d} x_{3}=-\mathrm{d}_{\perp} \Omega^{(2,0)}=\partial_{2} F^{-1} \mathrm{~d} x_{1} \wedge \mathrm{d} x_{2} \wedge \mathrm{d} x_{3} .
$$

This implies $\partial_{3} \mu_{1}-\partial_{1} \mu_{2}=\partial_{2} F^{-1}$ which is solved by, e.g., $\mu_{1}=\int \partial_{2} F^{-1} \mathrm{~d} x_{3}, \mu_{2}=0$ yielding

$$
\Omega=F\left(x_{1}, x_{2}, x_{3}\right)^{-1} \mathrm{~d} x_{1} \wedge \mathrm{d} x_{3}+\left(\int \partial_{2} F^{-1} \mathrm{~d} x_{3}\right) \mathrm{d} x_{1} \wedge \mathrm{d} x_{2}
$$

as compatible presymplectic form.

Note that $\Omega$ is well-defined globally even if $\omega$ is irrational in which case the leaves are dense in the torus factor of $M$. However, if we change $M$ to be $T^{2} \times S^{1}$, the $x_{3^{-}}$ integration in the second term of $\Omega$ is not periodic if there is a nonvanishing zero-mode in the Fourier decomposition of $\partial_{2} F^{-1}$ with respect to $x_{3}$. This means that in such a case the characteristic form class of $\Pi$ on $T^{2} \times S^{1}$ does not vanish. If $\omega$ is rational the leaves in $M$ have nontrivial second cohomology, whereas for irrational $\omega$ the leaves are of topology $\mathbb{R} \times S^{1}$ and so have trivial second cohomology, but $M$ is not foliated trivially. So in both cases, there is no contradiction to Lemma 6 .

\subsection{Leafwise symplectic embeddings of Poisson manifolds}

Theorem 1 asserts that any Poisson manifold $\left(P, \Pi_{P}\right)$ has a symplectic realization. This provides an appropriate Poisson map from a symplectic manifold to the given Poisson manifold. As demonstrated in Sec. 3 above (cf. in particular Proposition 2 and diagram (11)), physically this is, e.g., of interest in constrained systems with a closed algebra: Any Poisson manifold (or at least any region of it contained in $\mathbb{R}^{d}, d=\operatorname{dim} P$ ) can be

\footnotetext{
${ }^{12}$ We thank A. Weinstein for suggesting to look at such an example.
} 
understood as arising from a constrained Hamiltonian system with appropriate constraint $\operatorname{map} \phi$.

The Dirac bracket and the considerations of the present section motivate the question for another map, going in a reverse direction. Clearly, the identity map from $(S, \omega)$ to $\left(S, \Pi_{D}\right)$ is not a Poisson map (here $\Pi_{D}$ is the Dirac bivector (16) defined for some second class constraints; $S$ is the neighborhood of $C$ for which $\Pi_{D}$ exists). Still, $\Pi_{D}$ and $\omega$ are by no means unrelated; they are what we called compatible to one another. In the language of maps, this may be rephrased as follows: The embedding map from $\left(S, \Pi_{D}\right)$ into $(M, \omega)$ is leafwise symplectic.

Definition 11 A map from a Poisson manifold $\left(P, \Pi_{P}\right)$ to a symplectic manifold $(M, \omega)$ is leafwise symplectic (or leaf-symplectic), if its restriction to any symplectic leaf of $\left(P, \Pi_{P}\right)$ is a symplectic map.

Remark: Recall that according to Def. 3 a map $f$ between two symplectic manifolds $\left(M_{1}, \omega_{1}\right)$ and $\left(M_{2}, \omega_{2}\right)$ is symplectic, iff $f^{*} \omega_{2}=\omega_{1}$. Therefore $f:\left(P, \Pi_{P}\right) \rightarrow(M, \omega)$ is leaf-symplectic, iff $\left(f \circ \iota_{L}\right)^{*} \omega=\Omega_{L}$ for any leaf $L$ of $\left(P, \Pi_{P}\right), \Omega_{L}$ being the induced symplectic 2-form on $L$.

Proposition 11 A regular Poisson manifold $\left(P, \Pi_{P}\right)$ permits a leaf-symplectic embedding into some symplectic manifold, iff its characteristic form-class vanishes.

Proof: According to Proposition 9, there exists a compatible presymplectic form on $P$ iff the characteristic form-class of $\Pi_{P}$ vanishes. Assuming that there exists a leaf-symplectic embedding $f: P \rightarrow M$ from $P$ to $(M, \omega), f^{*} \omega$ provides a compatible presymplectic form on $\left(P, \Pi_{P}\right)$. So the vanishing of the characteristic form-class is a necessary condition. On the other hand, if it vanishes, there exists a compatible presymplectic form $\Omega$ on $P$. According to Theorem 2, $(P, \Omega)$ can be embedded coisotropically into a symplectic manifold $(M, \omega)$. Denoting this embedding by $\iota$ and the embedding of a symplectic leaf $\left(L, \Omega_{L}\right)$ into $P$ by $\iota_{L}$, we have $\Omega_{L}=\iota_{L}^{*} \Omega=\iota_{L}^{*} \iota^{*} \omega=\left(\left.\iota\right|_{L}\right)^{*} \omega$. Thus the condition is also sufficient.

Leaf-symplectic embeddings can be regarded as a concept related to isotropic symplectic realizations ${ }^{[3}$, which are Poisson maps $r:(Y, \omega) \rightarrow(M, \Pi)$ from a symplectic manifold $(Y, \omega)$ to a Poisson manifold $(M, \Pi)$ such that the fibers of $r$ are isotropic (i.e. $T F \subset(T F)^{\perp}$ for any fiber $\left.F=r^{-1}(m), m \in M\right)$. They are of interest for symplectic groupoids [13, 37], which also appeared recently in the context of Poisson Sigma Models in Ref. [38]. Obstructions for the existence of an isotropic symplectic realization have been derived [39, 9] which are of cohomological nature and similar to those derived here for the existence of a leaf-symplectic embedding.

While the Dirac bracket, which provided the motivation for defining leaf-symplectic embeddings, is usually used only on symplectic manifolds $(M, \omega)$ there is a straightforward generalization of Def. 11 to the case of a Poisson target manifold:

\footnotetext{
${ }^{13}$ We are grateful to A. Weinstein for making us aware of this relation.
} 
Definition 12 A map from a Poisson manifold $\left(P_{1}, \Pi_{1}\right)$ to a Poisson manifold $\left(P_{2}, \Pi_{2}\right)$ is leaf-to-leaf symplectic if the image of any leaf $L_{1}$ of $P_{1}$ is symplectically embedded in a leaf $L_{2}$ of $P_{2}$.

Remarks: i) If $\left(P_{2}, \Pi_{2}\right)$ is symplectic, this clearly reduces to Def. 11 .

ii) Unlike leaf-symplectic embeddings, there always exists a leaf-to-leaf symplectic embedding of a Poisson manifold $(P, \Pi)$, namely the identity map.

iii) Non-trivial examples of leaf-to-leaf symplectic embeddings are given by second class submanifolds of a Poisson manifold as defined in part (iii), (b) of Def. 2 (see also the discussion following this definition). Other examples are cosymplectic submanifolds [8] and Dirac submanifolds [40] of a Poisson manifold; this follows from Corollary 2.11 and Theorem 2.3, (vi) of [40].

The situation in Def. 12 corresponds to a (generalized) Dirac bracket constructed for a family of second class submanifolds $C$ in a (nonsymplectic) Poisson manifold $(P, \Pi)$ in the following way: We use the notation of Remark (iii) at the end of Sec. 4.1. However, for a degenerate Poisson structure $\Pi$ there is no symplectically orthogonal complement of $T_{x} C$. Instead, we use the fact that $C$ is, as a consequence of Def. 2, contained in a leaf $L$ of $P$ with symplectic structure $\Omega_{L}$ which defines the complement of $T_{x} C$ in $T_{x} L=T_{x} C \oplus T_{x} C^{\perp}$. Similarly, we have $T_{x}^{*} L=\operatorname{Ann}_{L}\left(T_{x} C^{\perp}\right) \oplus \operatorname{Ann}_{L}\left(T_{x} C\right)$. The projection $\bar{\pi}_{1}$ is now defined in two steps: we first project $T_{x}^{*} P$ to $T_{x}^{*} P / \operatorname{Ann}_{P}\left(T_{x} L\right) \cong T_{x}^{*} L$, followed by a projection to the first factor in the decomposition of $T_{x}^{*} L$. Since $\operatorname{Ann}_{P}\left(T_{x} L\right)=\operatorname{ker} \Pi_{x}^{\sharp}$ the Poisson bivector factors through the first projection and $\Pi_{D}=\Pi \circ\left(\bar{\pi}_{1} \otimes \bar{\pi}_{1}\right)$ defines a bivector which generalizes the Dirac bracket.

\section{Acknowledgements}

We thank A. Alekseev, M. Bordemann, P. Bressler and in particular A. Weinstein for interesting discussions and suggestions, and L. Dittmann for help with drawing the diagrams. M. B. is grateful for support from NSF grant PHY00-90091 and the Eberly research funds of Penn State, and to A. Wipf and the TPI in Jena for hospitality during an essential part of the completion of this work. T. S. thanks the Erwin Schrödinger Institute in Vienna for hospitality during an inspiring workshop on Poisson geometry.

\section{References}

[1] P.A.M. Dirac, Lectures on Quantum Mechanics, Yeshiva University, New York: Academic Press, 1967.

[2] M. Kontsevich, Deformation quantization of Poisson manifolds, I, q-alg/9709040.

[3] A. Cannas da Silva and A. Weinstein, Geometric models for noncommutative algebras, American Mathematical Society (AMS). Providence, RI, 1999. 
[4] I. Vaisman, On the geometric quantization of Poisson manifolds, J. Math. Phys. 32 (1991) 3339-3345.

[5] V. Schomerus, D-branes and Deformation Quantization, JHEP 9906 (1999) 030

[6] B. Jurco, P. Schupp, J. Wess, Noncommutative gauge theory for Poisson manifolds, Nucl.Phys. B584 (2000) 784-794.

[7] A. Y. Alekseev, V. Schomerus, and T. Strobl, Closed constraint algebras and path integrals for loop group actions, J. Math. Phys. 42 (2001) 2144-2155.

[8] A. Weinstein, The local structure of Poisson manifolds, J. Diff. Geom. 18 (1983) 523557.

[9] I. Vaisman, Lectures on the Geometry of Poisson Manifolds, Birkhäuser, Basel, 1994.

[10] R. A. Bertlmann, Anomalies in Quantum Field Theory, Clarendon Press, Oxford, 1996.

[11] G. Barnich, F. Brandt, and M. Henneaux, Local BRST cohomology in gauge theories, Phys. Rept. 338 (2000) 439-569.

[12] M. Karasev, Analogues of objects of the theory of Lie groups for nonlinear Poisson brackets, Math. USSR Izvestiya 28 (1987) 497-527.

[13] A. Weinstein, Symplectic Groupoids and Poisson Manifolds, Bull. Am. Math. Soc. 16 (1987) 101-104.

[14] M. J. Gotay, On coisotropic embeddings of presymplectic manifolds, Proc. Am. Math. Soc. 84 (1982) 111-114.

[15] V. Guillemin and S. Sternberg, Symplectic Techniques in Physics, Cambridge University Press, Cambridge, 1984.

[16] L. D. Faddeev and S. L. Shatashvili, Realization of the Schwinger term in the Gauss law and the possibility of correct quantization of a theory with anomalies, Phys. Lett. B 167 (1986) 225-228.

[17] I. A. Batalin and E. S. Fradkin, Operatorial quantization of dynamical systems subject to second class constraints, Nucl. Phys. B 279 (1987) 514-528.

[18] I. A. Batalin, M. A. Grigoriev, S. L. Lyakhovich, Star Product for Second Class Constraint Systems from a BRST Theory, hep-th/0101089.

[19] M. Bojowald and T. Strobl, Classical Solutions for Poisson Sigma Models on a Riemann surface, in preparation. 
[20] T. Strobl, Gravity in two spacetime dimensions, Habilitation thesis, RWTH Aachen, June 1999, hep-th/0011240.

[21] P. Schaller and T. Strobl, Poisson structure induced (topological) field theories, Mod. Phys. Lett. A9 (1994) 3129-3136, hep-th/9405110.

[22] N. Ikeda, Two-Dimensional Gravity and Nonlinear Gauge Theory, Ann. Phys. 235 (1994) 435-464.

[23] I. Vaisman, Symplectic Geometry and Secondary Characteristic Classes, Birkhäuser, Basel, 1987.

[24] T.J. Courant, Dirac manifolds, Trans. A.M.S. 319 (1990) 631-661.

[25] Z.J. Liu, A. Weinstein and P. Xu, Manin triples for Lie bialgebroids, J. Diff. Geom. 45 (1997) 547-574.

[26] R. U. Sexl and H. K. Urbantke, Relativity, Groups, Particles: Special Relativity and Relativistic Symmetry in Field and Particle Physics, Springer-Verlag, New York, 2001.

[27] A. Weinstein, Coisotropic calculus and Poisson groupoids, J. Math. Soc. Japan 40 (1988) 705-727.

[28] R. Bott and L. W. Tu, Differential forms in algebraic topology, Graduate Texts in Mathematics 82, Springer-Verlag, New York, 1991.

[29] V.N. Gribov, Quantization of nonabelian gauge theories, Nucl. Phys. B139 (1978) 1.

[30] M. Henneaux and C. Teitelboim, Quantization of gauge systems, Princeton University Press, Princeton, 1992.

[31] S. Lyakhovich and R. Marnelius, Extended observables in theories with constraints, hep-th/0105099.

[32] C. Klimčík and T. Strobl, WZW-Poisson manifolds, math.SG/0104189.

[33] J.-S. Park, Topological Open P-Branes, hep-th/0012141.

[34] P. Severa and A. Weinstein, Poisson geometry with a 3-form background, math.SG/0107133.

[35] T. Strobl, in preparation.

[36] J.E. Marsden and T.S. Ratiu, Introduction to Mechanics and Symmetry, 2nd edition, Springer, New York, 1999.

[37] M. V. Karasev and V. P. Maslov, Nonlinear Poisson Brackets: Geometry and Quantization, Translations of Mathematical Monographs 119, AMS, Providence, 1993. 
[38] A. S. Cattaneo and G. Felder, Poisson sigma models and symplectic groupoids, math.SG/0003023.

[39] D. Dazord and T. Delzant, Le problème général des variables actions-angles, J. Diff. Geom. 26 (1987), 223-251.

[40] P. Xu, Dirac submanifolds and Poisson involutions, math.SG/0110326. 